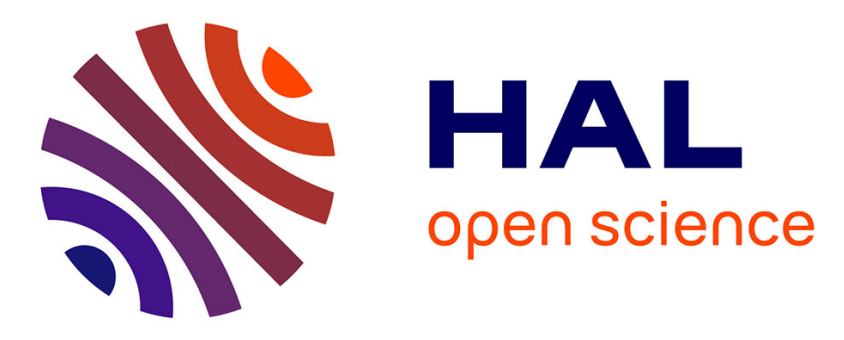

\title{
Photorespiratory serine hydroxymethyltransferase 1 activity impacts abiotic stress tolerance and stomatal closure
}

Yanpei Liu, Caroline Mauve, Marlène Lamothe-Sibold, Florence Guérard, Nathalie Glab, Michael Hodges, Mathieu Jossier

\section{To cite this version:}

Yanpei Liu, Caroline Mauve, Marlène Lamothe-Sibold, Florence Guérard, Nathalie Glab, et al.. Photorespiratory serine hydroxymethyltransferase 1 activity impacts abiotic stress tolerance and stomatal closure. Plant, Cell and Environment, 2019, 42 (9), pp.2567 - 2583. 10.1111/pce.13595 . hal03094148

\author{
HAL Id: hal-03094148 \\ https://hal.science/hal-03094148
}

Submitted on 21 Jul 2021

HAL is a multi-disciplinary open access archive for the deposit and dissemination of scientific research documents, whether they are published or not. The documents may come from teaching and research institutions in France or abroad, or from public or private research centers.
L'archive ouverte pluridisciplinaire HAL, est destinée au dépôt et à la diffusion de documents scientifiques de niveau recherche, publiés ou non, émanant des établissements d'enseignement et de recherche français ou étrangers, des laboratoires publics ou privés. 
1 Photorespiratory serine hydroxymethyltransferase 1 activity impacts abiotic stress tolerance and stomatal closure

3

4 Yanpei Liu, Caroline Mauve, Marlène Lamothe-Sibold, Florence Guérard, Nathalie Glab, 5 Michael Hodges, Mathieu Jossier*

Institute of Plant Sciences Paris-Saclay, CNRS, Université Paris-Sud, INRA, Université d'Evry, Université Paris-Diderot, Université Paris-Saclay, 91405 Orsay Cedex, France

*Corresponding author: Tel. +33 169153335; e-mail: mathieu.jossier@ips2.universite-paris$\underline{\text { saclay.fr }}$

\section{Abstract}

The photorespiratory cycle is a crucial pathway in photosynthetic organisms since it removes toxic 2-phosphoglycolate made by the oxygenase activity of ribulose-1,5-bisphosphate carboxylase/oxygenase and retrieves its carbon as 3-phosphoglycerate. Mitochondrial serine hydroxymethyltransferase 1 (SHMT1) is an essential photorespiratory enzyme converting glycine to serine. SHMT1 regulation remains poorly understood although it could involve the phosphorylation of serine 31. Here we report the complementation of Arabidopsis thaliana shm1-1 by SHMT1 wild-type, phosphorylation mimetic (S31D) or non-phophorylatable (S31A) forms. All SHMT1 forms could almost fully complement the photorespiratory growth phenotype of shm1-1, however each transgenic line had only $50 \%$ of normal SHMT activity. In response to either a salt or drought stress, Compl-S31D lines showed a more severe growth deficiency compared to the other transgenic lines. This sensitivity to salt appeared to reflect reduced SHMT1-S31D protein amounts and a lower activity that impacted leaf metabolism leading to proline under-accumulation and over-accumulation of polyamines. The S31D mutation in SHMT1 also led to a reduction in salt-induced and ABA-induced stomatal closure. Taken together, our results highlight the importance of maintaining photorespiratory SHMT1 activity in salt and drought stress conditions and indicate that SHMT1 S31 phosphorylation could be involved in modulating SHMT1 protein stability. 
30 Keywords: Photorespiration, Protein phosphorylation, SHMT1, Metabolism, Abiotic stress, 31 stomata

32

33 


\section{Introduction}

Photorespiration is a key primary metabolic pathway that starts with the fixation of $\mathrm{O}_{2}$ through the oxygenase activity of ribulose-1,5-bisphosphate carboxylase/oxygenase (RuBisCO) leading to the synthesis of one 2-phosphoglycolate (2PG) and one 3phosphoglycerate (3PGA) (Bauwe et al., 2010). However, 2PG, and other intermediates of photorespiration such as glycolate have been shown to reduce photosynthesis by inhibiting triose-phosphate isomerase and RuBisCO, respectively (Anderson, 1971; Kelly and Latzko, 1976; Flügel et al., 2017). Therefore, the photorespiratory cycle is crucial to remove these toxic metabolites and to recover the carbon as 3PGA. It takes place in four cell compartments (cytosol, chloroplasts, peroxisomes and mitochondria) and involves eight core enzymes and several transporters. Together they convert two molecules of 2PG into one molecule of 3PGA, and in doing so consume one molecule of NADH and ATP and produce one molecule of $\mathrm{CO}_{2}$, $\mathrm{NH}_{3}, \mathrm{NADH}$ and ADP (Bauwe et al., 2010; see Figure 1). The photorespiratory cycle interacts with a number of primary metabolic processes such as photosynthesis, nitrate and amino acid metabolism, the tricarboxylic acid (TCA) cycle, and C1-metabolism (Takahashi et al., 2007; Tcherkez et al., 2008; Hanson and Gregory, 2011; Ros et al., 2014; Bloom, 2015). It also impacts pathogen defense (Sørhagen et al., 2013) and more recently it has been proposed to affect stomatal aperture (Eisenhut et al., 2017). The importance of the photorespiratory cycle is highlighted by the strong phenotypes exhibited by air-grown knock-out mutants of photorespiratory enzymes and transporters that include reduced growth, chlorosis, and even lethality. Although the phenotypic severity depends on the affected protein, all photorespiratory mutants can be rescued under high $\mathrm{CO}_{2}$ growth conditions (Timm and Bauwe, 2013).

Amongst the first photorespiratory cycle mutants identified from ethyl methanesulfonate (EMS)-mutated Arabidopsis thaliana were three allelic stm (later renamed shm) mutants affected in mitochondrial serine hydroxymethyl transferase (EC 2.1.2.1; SHMT) activity (Somerville and Ogren, 1981). In A. thaliana, two of the seven putative SHMT genes appear to encode mitochondrial SHMT isoforms (AtSHMT1, At4g37930; AtSHMT2, At5g26780) (McClung et al., 2000; Engel et al., 2011). The shm1-1 (CS8010) mutant of Somerville and co-workers was mapped to the SHMT1 gene (At4g37930) (Voll et al., 2006). It displays a strong-to-intermediate photorespiratory phenotype that is rescued under high $\mathrm{CO}_{2}$ (Timm and Bauwe, 2013). This phenotype shows that SHMT1 has a non-redundant photorespiratory role in which it catalyzes the transfer of a hydroxymethyl group from 5,10-methylene-THF, 
produced by the glycine decarboxylase complex (GDC), to a glycine to form serine (Figure 1). Interestingly, a shm1-1 x shm2-1 double mutant lacking both mitochondrial SHMT isoforms was unable to survive even in $\mathrm{CO}_{2}$-enriched air probably because of the impairment of the additional role of SHMT in C1-metabolism (Engel et al., 2011).

Photorespiratory enzymes also appear to play important roles in plant responses to biotic and abiotic stresses including high temperature, drought, salinity and pathogen attack (Moreno et al., 2005; Rojas et al., 2012; Li and Hu, 2015; Noctor et al., 2015; Cui et al., 2016; Xu et al., 2018). In photosynthetic bacteria, the overexpression of SHMT from the halotolerant cyanobacteria Aphanothece halophytica either in E. coli or in the fresh water cyanobacteria Synechococcus elongates conferred a better tolerance to salinity (Waditee-Sirisattha et al., 2012, 2016). In plants, SHMT1 is essential in response to pathogens and it was also suggested to be involved in salt tolerance (Moreno et al., 2005). Recently, a salt-tolerant rice variety was found to have a high SHMT expression associated to a strong accumulation of serine in response to salt stress when compared to a salt-sensitive variety (Mishra et al., 2016).

Although SHMT1 has a key role in the photorespiratory cycle and it has been shown to be involved in abiotic and biotic stress responses, the regulation of SHMT1 activity remains to be elucidated. To rapidly respond to changing conditions, enzymes are often posttranslationally modified and several reports suggest that this could be the case for SHMT1. Indeed, SHMT1 is a potential target of thioredoxin-dependent redox regulation (Marchand et al., 2004). SHMT1 was also found to be degraded in a 26S proteasome-dependent process, and ubiquitin-specific protease 16 (UBP16) stabilized SHMT1 during a salt stress by removing conjugated ubiquitin (Zhou et al., 2012). Pea mitochondrial SHMT was found to be nitrosylated under control conditions but not during a salt stress (Camejo et al., 2013). According to the Arabidopsis phosphopeptide database PhosPhAt (http://phosphat.unihohenheim.de/), 8 residues of SHMT1 are phosphorylated. Previously, we reported that the phosphorylation of serine 31 (S31) of the phosphopeptide (pS)LPSEAVDEKER remained unchanged in Arabidopsis rosettes when treated with varying $\mathrm{O}_{2} / \mathrm{CO}_{2}$ conditions suggesting that this phosphorylation event was not correlated to leaf photorespiratory activity (Abadie et al., 2016). It should be noted that after removal of the mitochondrial signal peptide and a further processing by intermediate cleavage peptidase 55 (ICP55; Huang et al., 2015), S31 become $\mathrm{S} 1$ of the mature SHMT1 protein.

In this work, the function of Arabidopsis thaliana SHMT1 S31 phosphorylation was investigated by studying shm1-1 plants complemented with either wild-type, phosphorylation 
mimetic (S31D) or non-phophorylatable (S31A) SHMT1. In normal air-grown conditions, no significant differences in growth were observed between the transgenic lines, although they were smaller than WT Col-0 plants. However, when subjected to either a salt or a drought stress, Compl-S31D lines exhibited a further reduction in growth. In the case of a salt treatment, the increased sensitivity was associated with altered SHMT1-S31D protein and activity levels. Our data will be discussed in terms of the importance of photorespiratory SHMT1 activity in plant abiotic stress tolerance and ABA-induced stomatal movements, and the possible role of S31 phosphorylation in modulating SHMT1 protein stability.

\section{Materials and methods}

\section{Plants material and growth conditions}

Arabidopsis thaliana Columbia (Col-0) accession was used as a wild-type control. The shml1 mutant (Somerville and Ogren, 1981; Voll et al, 2006) was provided by Andreas P.M. Weber (University of Dusseldorf, Germany).

For in vitro experiments, sterilized seeds were sown on $1 / 2 \mathrm{MS}$ medium (Murashige and Skoog, 1962), supplemented with $0.8 \%$ agar (w/v), and placed at $4{ }^{\circ} \mathrm{C}$ in the dark for $48 \mathrm{~h}$ to promote germination. Plants were grown in short-day conditions (8h light/16h dark, $160 \mu \mathrm{mol}$ photons. $\mathrm{m}^{-2} \cdot \mathrm{s}^{-1}, 65 \%$ humidity, $20^{\circ} \mathrm{C}$ day $/ 18^{\circ} \mathrm{C}$ night, ambient air $\mathrm{CO}_{2}$ concentration of around $\left.400 \mu \mathrm{L} . \mathrm{L}^{-1}\right)$. For in vitro salt treatments, one-week-old seedlings were grown vertically on $1 / 2 \mathrm{MS}$-agar plates before their transfer to new plates with or without $50 \mathrm{mM} \mathrm{NaCl}$ or 100 $\mathrm{mM}$ mannitol according to Jossier et al., 2010. For soil-grown experiments, Arabidopsis seeds were germinated on potting soil for 10 days in short days (8h light/16h dark, $200 \mu \mathrm{mol}$ of photons. $\mathrm{m}^{-2} . \mathrm{s}^{-1}, 65 \%$ humidity, $20^{\circ} \mathrm{C}$ day $/ 18^{\circ} \mathrm{C}$ night, ambient air $\mathrm{CO}_{2}$ ). Subsequently, seedlings were transplanted to individual pots and grown further in short day conditions either in ambient air $\mathrm{CO}_{2}$ or high $\mathrm{CO}_{2}\left(3000 \mu \mathrm{L} . \mathrm{L}^{-1}\right)$ enriched air $(\mathrm{HC})$. For the drought treatment, 10-day-old Arabidopsis seedlings were transplanted and grown for 2 weeks in ambient air $\mathrm{CO}_{2}$ short-day-conditions before being subjected to water deprivation for 3 weeks then rewatering for 3 days.

\section{Plasmid construction, site-directed mutagenesis and generation of complemented lines}

Genomic DNA was extracted from A. thaliana Col-0 leaves according to Edwards et al. (1991), and the SHMT1 promoting region (-1000 to +55) was PCR-amplified using primers (promSHMT1-F/promSHMT1-R). Note, all primers are described in Supplemental Table S4. 
The resulting DNA (SHMT1 promoter) was cloned into pGEM-T Easy® and sequenced (by MWG OPERON, EUROFINS). The SHMT1 cDNA in pUNI51 (clone U21423) was obtained from the Arabidopsis Biological Resource Center (ABRC) (https://abrc.osu.edu/). SHMT1 cDNA was PCR-amplified using primers cDNA-SHMT1-F and GW-SHMT1-R and the SHMT1 promoter region was re-amplified with the primers described above. The two resulting DNA fragments (SHMT1 cDNA and SHMT1 promoter), with an overlapping region $(0$ to +55$)$, were used as templates for two consecutive PCR amplifications (using primers GW-SHMT1-F/GW-SHMT1-R followed by GateWay-F/GateWay-R) in order to join the two DNAs and to add attB Gateway ${ }^{\circledR}$ recombination sites to the 5' and 3' ends. The resulting DNA (promSHMT1::SHMT1) was further sequenced before being cloned into pDONR207 using the GATEWAY® cloning system to generate pDONR207-promSHMT1::SHMT1.

To introduce the required point mutations into pDONR207-promSHMT1::SHMT1, primer pairs were selected and used with the QUIKCHANGE® II XL Site-Directed Mutagenesis Kit (AGILENT®), according to the manufacturer's instructions to obtain pDONR207promSHMT1::SHMT1-S31A and pDONR207-promSHMT1::SHMT1-S31D. Then, promSHMT1::SHMT1, promSHMT1::SHMT1-S31A and promSHMT1::SHMT1-S31D were subcloned into pH7WG using the GATEWAY® cloning system to generate the plant transformation constructs pH7WG-promSHMT1::SHMT1, pH7WG-promSHMT1::SHMT1S31A and pH7WG-promSHMT1::SHMT1-S31D. They were used to transform HC-grown shm1-1 plants by Agrobacterium-mediated floral dip (Clough and Bent, 1999). Hygromycinresistant seeds were selected and homozygous transgenic lines containing a single insertion were selected by successive rounds of self-crossing and antibiotic selection on $1 / 2 \mathrm{MS}$ medium agar plates.

\section{Analysis of N-terminal sequence of SHMT1 protein}

Arabidopsis rosette leaves (100 mg FW) were rapidly frozen in liquid nitrogen and stored at $80^{\circ} \mathrm{C}$ until extraction. Protein extraction, LC-MS/MS analysis and identification of SHMT1 peptides were carried out as previously described (Boex-Fontvieille et al., 2013).

\section{Leaf gas exchange and chlorophyll fluorescence measurements}

Leaf gas exchange and chlorophyll fluorescence measurements were carried out on $2 \mathrm{~cm}^{2}$ of fully expanded leaves of 6-week-old plants in a gas exchange chamber (LCF 6400-40, LiCOR) connected to a portable measuring system (LI 6400XT, LiCOR) (Dellero et al., 2015). Standard measuring conditions were: $200 \mu \mathrm{mol}$ photons $\mathrm{m}^{-2} \mathrm{~s}^{-1}$ of light intensity, a leaf 
temperature of $21^{\circ} \mathrm{C}, 60-70 \%$ relative humidity [vapor pressure deficit (VPD) of the leaf approximately 1], $400 \mu \mathrm{mol} \mathrm{mol}^{-1} \mathrm{CO}_{2}$ and $21 \% \mathrm{O}_{2}$. For chlorophyll fluorescence parameters, plants were adapted for $30 \mathrm{~min}$ to darkness before measuring the Fo and Fm levels. Parameters were calculated as follows: $\mathrm{Fv}=\mathrm{Fm}-\mathrm{Fo}, \mathrm{NPQ}=\left(\mathrm{Fm}-\mathrm{Fm}^{\prime}\right) / \mathrm{Fm}^{\prime}, \mathrm{ETR}=\left[\left(\mathrm{Fm}^{\prime}-\right.\right.$ $\left.\mathrm{Fv}) / \mathrm{Fm}^{\prime}\right] 0.5 \times \mathrm{I} \times \alpha$ leaf, with I corresponding to the irradiance in $\mu \mathrm{mol}$ photons $\mathrm{m}^{-2} \mathrm{~s}^{-1}$ and $\alpha$ leaf to the light absorption coefficient of a leaf $=0.85$.

\section{SHMT activity assays}

SHMT activity was determined by following the production of glycine from serine as measured by HPLC. $400 \mathrm{mg}$ of rosette or seedling leaves were harvested and ground to a fine powder in liquid nitrogen. Total soluble protein was extracted in $500 \mu \mathrm{l}$ buffer containing 50 $\mathrm{mM} \mathrm{NaH} \mathrm{PO}_{4}$, anti-protease cocktail (Complete-Mini Free-EDTA, ROCHE ${ }^{\circledR}$ ), $1 \mathrm{mM} \beta$ mercaptoethanol, $\mathrm{pH}$ 8.0. Extracts were clarified by centrifugation at $16000 \mathrm{xg}$ for $30 \mathrm{~min}$ at $4^{\circ} \mathrm{C}$, and the resulting supernatant was desalted by NAP-5 (HEALTHCARE) size-exclusion filtration after equilibration with $50 \mathrm{mM} \mathrm{NaH} \mathrm{PO}_{4}, \mathrm{pH}$ 8.0. Protein concentration was determined using Bradford reagent (B6916, SIGMA-ALDRICH). Each reaction was composed of $50 \mathrm{mM} \mathrm{NaH} \mathrm{PO}_{4}, \mathrm{pH} 8.0,0.25 \mathrm{mM}$ pyridoxal-5-phosphate, $2 \mathrm{mM} \mathrm{5,10-}$ methylene tetrahydrofolate, $20 \mathrm{mM}$ serine, $0.15 \mathrm{mM} \alpha$-amino-n-butyric acid, $400 \mu \mathrm{g}$ of total soluble protein in a final volume of $500 \mu \mathrm{l}$ and incubated at $25^{\circ} \mathrm{C}$ for $5 \mathrm{~min}$ (determined to be in the linear phase of the reaction). The reaction was stopped by adding $175 \mu \mathrm{L}$ of trichloroacetic acid $25 \%(\mathrm{v} / \mathrm{v})$ followed by incubation on ice for $15 \mathrm{~min}$. After centrifugation at $15000 \mathrm{xg}$ for $10 \mathrm{~min}$ at $4^{\circ} \mathrm{C}, 480 \mu \mathrm{L}$ of the supernatant were transferred to a new tube, the $\mathrm{pH}$ was neutralized with $50 \mu \mathrm{L}$ of $\mathrm{K}_{2} \mathrm{CO}_{3}$ buffer $\left(2.3 \mathrm{M} \mathrm{K}_{2} \mathrm{CO}_{3}, 50 \mathrm{mM} \mathrm{NaH} \mathrm{PO}_{4}, \mathrm{pH} 8.0\right.$ ) and glycine was quantified by HPLC (see below).

\section{RNA isolation and real-time RT-qPCR}

Two-week-old Arabidopsis seedlings were transferred to liquid 1/2MS medium containing 50 $\mathrm{mM} \mathrm{NaCl}$ for $6 \mathrm{~h}$ under light then gene expression was determined by RT-qPCR. Total RNA was extracted with the NucleoSpin RNA Plus Kit then $2 \mu \mathrm{g}$ RNA was used to make cDNA by reverse transcription (IM-PROMII ${ }^{\mathrm{TM}}$ ), according to the manufacturer's instructions. Quantitative real-time PCR was carried out with a LightCycle-480-Real-Time PCR System and SYBR green (ROCHE DIAGNOSTICS). Relative-expression levels were normalized to Actin-2 (At3g18780) and three independent experiments were performed. Primers are given in Supplemental Table S4. 


\section{Amino acid analyses by HPLC and metabolite analyses by GC-MS}

199 Seven-day-old in vitro grown seedlings were transferred to $1 / 2$ MS plates with or without 50 $\mathrm{mM} \mathrm{NaCl}$ for 3 weeks, rosette leaves (100 mg FW) were then collected after 5 hours of light and rapidly frozen in liquid nitrogen and stored at $-80^{\circ} \mathrm{C}$ until extraction. Quantitative glycine amounts were measured by HPLC and relative metabolite levels of whole rosette leaves were analyzed by GC-MS, both after cold-methanol extraction, as described in Noctor et al. (2007).

\section{SDS-PAGE and western-blot}

206

Ten $\mu \mathrm{g}$ of soluble proteins were separated on an SDS-PAGE gel (10\% acrylamide) and they were detected by Coomassie blue staining (Laemmli, 1970). For western-blot analyses, SDSPAGE separated proteins were transferred to nitrocellulose membranes, blocked for $1 \mathrm{~h}$ at room temperature in blocking buffer (5\% non-fat dry milk in TBST buffer including $50 \mathrm{mM}$ Tris-base, $150 \mathrm{mM} \mathrm{NaCl}, 0.1 \%$ Tween 20, $\mathrm{pH} 7.4$ ) and incubated overnight at $4{ }^{\circ} \mathrm{C}$ with either a 1/3500 dilution (in blocking buffer) of potato SHMT1 antibodies (Engel et al., 2011) or actin antibodies (A0480, SIGMA-ALDRICH) diluted 1/5000. Membranes were washed 3times with TBST buffer, then incubated with either 1/5000 diluted anti-rabbit (A6154, SigmaAldrich) or anti-mouse antibodies (A4416, SIGMA-ALDRICH) for $1 \mathrm{~h}$ at room temperature in blocking buffer. Proteins were revealed using a chemiluminescent peroxidase substrate-3 kit (CPS350, SIGMA-ALDRICH).

\section{Analysis of $\mathrm{H}_{2} \mathrm{O}_{2}$ levels}

Hydrogen peroxide was measured by two methods. Seedlings were grown on $1 / 2 \mathrm{MS}$ plates for 14 days in short-day conditions before a $150 \mathrm{mM} \mathrm{NaCl}$ treatment $(14 \mathrm{~h})$ as in Zhou et al., 2012. For 3,3'-diaminobenzidine (DAB) staining, leaves were incubated in DAB staining buffer $\left(50 \mathrm{mg}\right.$ DAB dissolved in $45 \mathrm{~mL}$ sterile $\mathrm{H}_{2} \mathrm{O}$, with $1 \mathrm{M} \mathrm{HCl}$ to dissolve the $\mathrm{DAB}$, then $25 \mu \mathrm{l}$ Tween $20(\mathrm{v} / \mathrm{v})$ and $2.5 \mathrm{~mL}$ of $200 \mathrm{mM} \mathrm{Na}_{2} \mathrm{HPO}_{4}(\mathrm{pH}$ 6.8) were added to adjust the $\mathrm{pH}$ to 5.0). DAB staining buffer was removed after $8 \mathrm{~h}$ and replaced with a bleaching solution of ethanol:acetic acid:glycerol ( $\mathrm{v}: \mathrm{v}: \mathrm{v}=3: 1: 1)$. Bleaching was carried out in a boiling water bath for $15 \mathrm{~min}$ followed by $30 \mathrm{~min}$ under shaking at room-temperature. For $\mathrm{H}_{2} \mathrm{O}_{2}$ quantification, seedlings were treated as above but $\mathrm{H}_{2} \mathrm{O}_{2}$ content was measured by a colorimetric assay using the Hydrogen Peroxide Assay Kit according to the manufacturer's instructions (ABCAM®). 


\section{Water content and water loss assays}

Water content was measured using leaves of 3-week-old seedlings by comparing the fresh and dry weights based on the equation: water content $(\%)=100 \times(\mathrm{FW}-\mathrm{DW}) / \mathrm{FW}$. DW was measured after lyophilization in a Christ Alpha 2-4 (PCR-EN7049) for $72 \mathrm{~h}$. Water loss assays were carried out in a growth chamber where rosettes of six-week-old plants were cut and weighed at different times. Water loss is presented as the percentage of the initial FW at the indicated time.

\section{Stomatal aperture assays}

Stomatal aperture was measured according to Merlot et al. (2007). Briefly, for ABA-induced stomatal closure, leaves of 5-6 week-old plants were harvested in darkness at the end of the night period. The abaxial side of leaves were glued to a coverslip using non-toxic medical adhesive (Hollister Medical Adhesif (7730 NEWPHARMA)). To obtain isolated epidermal cells, leaves were peeled gently and coverslips with glued epidermis were placed in $4.5 \mathrm{~cm}$ diameter petri-dishes containing $50 \mathrm{mM} \mathrm{KCl}, 0.1 \mathrm{mM} \mathrm{CaCl}_{2}, 10 \mathrm{mM}$ MES-KOH, pH 6 for $1 \mathrm{~h}$ in the dark to ensure closed stomata. Then they were incubated under light $\left(200 \mu \mathrm{mol} . \mathrm{m}^{-2} . \mathrm{s}^{-1}\right)$ for $4 \mathrm{~h}$ to ensure stomatal opening, then incubated under light for $4 \mathrm{~h}$ in the presence/absence of $50 \mu \mathrm{M}$ ABA. Photographs of stomata were made using a microscope DMI 6000 (LEICA). Stomatal pore area (in $\mu^{2}$ ) was measured using ImageJ software. For the $\mathrm{NaCl}$-induced stomatal closure assay, plants were exposed to light $\left(200 \mu \mathrm{mol} . \mathrm{m}^{-2} . \mathrm{s}^{-1}\right)$ for $2.5 \mathrm{~h}$ before being watered with or without $300 \mathrm{mM} \mathrm{NaCl}$ for $4 \mathrm{~h}$ under light according to Chung et al., 2013. Leaves were harvested and stomatal aperture determined using isolated epidermal cells as described above. The same samples were used to determine stomatal index and stomatal density. For the study of stomatal behavior during dark to light and light to dark transitions, leaves were collected from plants two hours before the photoperiod then plants were exposed to light $\left(200 \mu \mathrm{mol} . \mathrm{m}^{-2} . \mathrm{s}^{-1}\right)$ for $4 \mathrm{~h}$ before being placed in the dark for $3 \mathrm{~h}$. Photographs were taken under a microscope AxioImager Z2 (ZEISS). Stomatal aperture (width/length) was measured using ImageJ software.

\section{Results}

Complementation of the shm1-1 photorespiratory growth phenotype by S31-mutated SHMT1 
To evaluate the impact of SHMT1 S31 phosphorylation on plant physiology and metabolism, site-directed mutagenesis was undertaken using the Arabidopsis cDNA of wild-type SHMT1, so that S31 was replaced either by a phosphomimetic aspartate (SHMT1-S31D) or by a nonphosphorylatable alanine (SHMT1-S31A) (Figure 2A). Subsequently, the resulting cDNAs were placed under the control of AtSHMT1 promoter region and used to transform high $\mathrm{CO}_{2^{-}}$ grown shml-1 plants to generate complemented plants named Compl-SWT, Compl-S31A and Compl-S31D (Figure 2B). Homozygous lines containing a single transgene insertion were selected for further analysis. While shm1-1 was unable to grow in air, this was not the case for the complemented lines although they were slightly smaller compared to wild-type (WT, Col0) control plants (Figure 2B). After 4 weeks on soil, the development of each transgenic line was similar when compared to each other (Figure 2B).

$\mathrm{N}$-terminal peptide analyses were performed by LC-MS/MS using extracts from rosettes of complemented lines (Compl-SWT-5, Compl-S31A-7 and Compl-S31D-5) to determine whether ICP55 processing was modified. In Compl-SWT-5 and Compl-S31A-7, the identified N-terminal peptides showed that ICP55 processing still took place however this was not the case for SHMT1 of Compl-S31D-5 since only a SDLPSEAVDEK peptide was observed (Supplemental Figure S1).

Western blot analyses were carried out to compare rosette leaf SHMT protein amounts between the different lines (Figure 2C). To allow the shm1-1 mutant to grow, plants were grown in high $\mathrm{CO}_{2}\left(3000 \mu \mathrm{L} . \mathrm{L}^{-1}\right)$. As expected, almost no SHMT protein was detected in shm1-1 rosettes while complemented lines exhibited different amounts of SHMT protein (between $40 \%$ and $70 \%$ of Col-0 SHMT protein levels) (Figure 2C and Supplemental Figure S2). Moreover, in accordance with SHMT protein levels in the complemented lines, SHMT activity analysis of high- $\mathrm{CO}_{2}$-grown rosette leaves showed that they all had a 40-60\% reduced activity compared to Col-0 (Figure 2D).

Since a reduced photorespiratory activity has a negative impact on photosynthetic $\mathrm{CO}_{2}$ assimilation (Somerville and Ogren, 1981; Chastain and Ogren, 1989; Timm et al., 2012) and electron transfer properties (Dellero et al., 2015, 2016), gas exchange and chlorophyll fluorescence parameters of fully expanded leaves were compared between 6-week-old WT plants and complemented lines. In the three transgenic lines Compl-SWT-5, Compl-S31D-5 and Compl-S31A-7, net $\mathrm{CO}_{2}$ assimilation rate $\left(A_{\mathrm{n}}\right)$ was reduced by $20 \%$ compared to control Col-0 plants while stomatal conductance $\left(g_{\mathrm{s}}\right)$, transpiration $(\mathrm{Tr})$, photosynthetic electron transfer rate (ETR), non-photochemical chlorophyll fluorescence quenching (NPQ) and 
Fv/Fm ratios were not significantly different (Supplemental Table S1). These results suggest that the smaller size of the complemented lines could be due to their reduced capacity to assimilate $\mathrm{CO}_{2}$ arising from their lower SHMT1 activity leading to an impaired photorespiratory cycle.

\section{Compl-S31D lines are hypersensitive to salt stress in air-grown conditions but not in}

\section{high $\mathrm{CO}_{2}$-growth conditions}

Since the different complemented lines did not show any distinct growth and photosynthetic differences between them, it was decided to test their response to abiotic stress. Since the shm1-3 mutant was found to be more sensitive to salt stress (Moreno et al., 2005), in vitro grown one-week-old WT, Compl-SWT-5, Compl-S31D-5 and Compl-S31A-7 seedlings were transferred to $50 \mathrm{mM} \mathrm{NaCl}$ or $100 \mathrm{mM}$ mannitol for 3 weeks. In the absence of $\mathrm{NaCl}$, no difference was observed between the complemented lines although they presented a growth deficiency compared to Col-0 seedlings (Figure 3A). This resulted in a 50\% decrease of seedling fresh weight (FW) (Figure 3B) and a 20\% decrease of primary root length (Supplemental Figure S3A). When transferred to mannitol, no significant differences were observed between the complemented lines but they all exhibited a $40 \%$ reduction of $\mathrm{FW}$ compared to Col-0 (Figure 3A and 3B). However, in the presence of $50 \mathrm{mM} \mathrm{NaCl}$, primary root length and seedling FW of the Compl-S31D-5 line showed significant reductions compared to Compl-S31A-7, Compl-SWT-5 and wild-type lines (Figure 3A and Supplemental Figure S3A). While Col-0 seedlings were slightly affected by the salt treatment, the Compl$S 31 D-5 \mathrm{FW}$ was decreased by $50 \%$ (Figure 3B) and primary root length was decreased by almost 30\% (Supplemental Figure S3A) when compared to Compl-S31A-7 and Compl-SWT-5 lines. Similar results were obtained for two other independent Compl-S31D lines (S31D-4 and $S 31 D-7)$ when treated with salt, both presenting a larger decrease in seedling FW and primary root length on 50mM NaCl (Supplemental Figure S4A and S4B). To determine whether the observed salt-induced growth phenotypes of Compl-S31D lines were associated with photorespiration, experiments were repeated in high $\mathrm{CO}_{2}$-growth conditions $\left(3000 \mu \mathrm{L}^{\mathrm{L}} \mathrm{L}^{-1}\right)$ and included the shm1-1 mutant as a control. After 4 weeks, Compl-S31D-5 seedlings did not display any differences in growth (seedling FW and primary root length) compared to shm1-1, Compl-SWT-5 and Compl-S31A-7 lines whether grown with or without $50 \mathrm{mM} \mathrm{NaCl}$ (Figure 3C and 3D, Supplemental Figure S3B). Again, these observations were confirmed with other Compl-S31D lines (Supplemental Figure S4C and S4D). Taken together, our data show that Compl-S31D lines are more sensitive to salt stress and this was photorespiration-dependent. 
329 Furthermore, the growth differences between air-grown Col-0 plants and the different transgenic lines also appear to be photorespiration-dependent whether under control or saltstress conditions (Figure 3).

\section{SHMT1 protein is less stable in the Compl-S31D-5 line in response to salt stress leading to a lower SHMT activity.}

To determine whether the salt-induced Compl-S31D-5 growth was linked to SHMT activity, this was measured using in vitro grown Col-0, Compl-SWT-5, Compl-S31D-5 and ComplS31A-7 lines under either control or a 3-week salt treatment (Figure 4A). Leaf total soluble proteins were extracted and SHMT activity was measured as the conversion of serine to glycine using an HPLC-based method (Simic et al., 2002). The salt treatment led to a significant $40 \%$ decrease in SHMT activity of Compl-S31D-5 while a much smaller decrease was measured for Compl-SWT-5 and Compl-S31A-7 (reduction of 13\% and 15\%, respectively) (Figure 4A). These activity changes appeared to mirror leaf SHMT protein levels as determined by western blot analyses (Figure 4B and 4C). Indeed, the SHMT protein level of Compl-S31D-5 was significantly reduced by $35 \%$ after a salt stress compared to Compl-SWT5 and Compl-S31A-7 (non-significant 11\% and 15\% reduction, respectively) (Figure 4B and 4C). Similar results were obtained for the other Compl-S31D lines (4 and 7) compared to the Compl-SWT (1 and 7) and Compl-S31A (4 and 6) lines (Supplemental Table S2). Taken together, it appeared that leaf SHMT1 protein of Compl-S31D-5 was less stable in response to salt leading to a lower SHMT activity, thus giving rise to a more severe salt-induced growth phenotype (Figure 3).

Metabolic responses of Col-0, Compl-SWT-5, Compl-S31A-7 and Compl-S31D-5 to salt stress

In order to explore the metabolic consequences of the reduced SHMT activity of Compl$S 31 D-5$ in response to salt stress, relative metabolite levels of whole rosettes of seedlings grown in vitro with or without $50 \mathrm{mM} \mathrm{NaCl}$ were analyzed by GC-MS (Supplemental Table S3). In control conditions, a limited number of similar differences were observed in all transgenic lines compared to Col-0. These included certain amino acids, organic acids and sugar levels. Indeed, asparagine, glutamate, glutamine, homoserine and tryptophan as well as the photorespiratory metabolites glycine, serine and glycerate accumulated in complemented lines (Figure 5). TCA cycle-associated organic acids including citrate, malate, and $\alpha$ ketoglutarate as well as glucose and fructose levels increased while dehydroascorbate 
decreased in complemented lines (Figure 5). In response to $50 \mathrm{mM} \mathrm{NaCl}$, while the relative levels of asparagine, glycine, glutamate and glutamine decreased in Col-0, Compl-SWT-5 and Compl-S31A-7, these amino acids remained at a higher level in Compl-S31D-5. On the other hand, levels of metabolites associated with stress protection such as proline, galactaric acid, and myo-inositol increased in all lines except Compl-S31D-5 in response to salt stress (Figure 5). Citrate levels did not change in response to salt stress in Compl-SWT-5 and Compl-S31A-7 but decreased in Compl-S31D-5. Interestingly, major metabolic differences concerned the levels of polyamines (spermidine and putrescine) and their precursors, ornithine and citrulline, that increased 3-fold (2-fold for putrescine) in response to salt only in Compl-S31D-5 (Figure 5). Hydrogen peroxide is a key element of stress-induced signaling pathways and Arabidopsis lacking SHMT1 exhibit a large accumulation of leaf $\mathrm{H}_{2} \mathrm{O}_{2}$ in response to salt stress (Moreno et al., 2005). In order to determine if the complemented lines exhibited altered leaf $\mathrm{H}_{2} \mathrm{O}_{2}$ contents, control and salt stressed Col-0, Compl-SWT-5, Compl-S31D-5 and Compl-S31A-7 seedlings where stained with DAB. The $\mathrm{H}_{2} \mathrm{O}_{2}$-induced brown deposit in Compl-S31D-5 was more important than the other plant lines after stress treatment indicating higher $\mathrm{H}_{2} \mathrm{O}_{2}$ levels while no differences were observed under control conditions (Figure 6A). A quantitative analysis of $\mathrm{H}_{2} \mathrm{O}_{2}$ content carried out on the same samples showed that all lines exhibited an increase in $\mathrm{H}_{2} \mathrm{O}_{2}$ after salt treatment, but the increase was 2-fold more important in Compl$S 31 D-5$ rosettes compared to the increase of other lines (Figure 6B and Supplemental Figure S5).

\section{Compl-S31D plants are unable to recover from a drought stress in ambient air}

Since our data indicated that Compl-S31D-5 plants were more sensitive to a salt stress, it was decided to test their sensitivity to drought. To achieve this, Col-0, Compl-SWT, Compl-S31A and Compl-S31D plants were grown on soil for 3 weeks in well-watered conditions, and then they were subjected to a drought stress by withholding water for 3 weeks before being rewatered. Under such conditions, the Compl-S31D lines were unable to recover from the drought stress (Supplemental Figure S6A). Moreover, detached leaves of Compl-S31D lines showed a faster initial water loss ratio compared to the other lines (Supplemental Figure S6B). It should be noted that all plant lines had similar initial water contents, (Supplemental Figure $\mathrm{S} 6 \mathrm{C})$.

\section{Compl-S31D stomata show a reduced sensitivity to closure in response to $\mathrm{NaCl}$ and $\mathrm{ABA}$}


In order to determine whether the observed stress sensitivity of the Compl-S31D-5 line was linked to impairment of stomatal development, stomata density and stomatal index of fiveweek-old plants grown on soil were measured, and no differences could be observed between all lines (Figure S7). As photorespiration occurs under light and is implicated in stomatal movements (Eisenhut et al., 2017), stomatal behavior of three-week-old plants grown on soil was tested during transitions from dark to light (after $4 \mathrm{~h}$ ) and light to dark (after $3 \mathrm{~h}$ ). Stomata of all lines exhibited a similar behavior for each tested transition (Supplemental Figure S8A). Stomatal closure was measured also in Col-0, Compl-SWT-5, Compl-S31D-5 and Compl-S31A-7 plants in response to salt treatment. Five-week-old soil-grown plants were exposed to a $4 \mathrm{~h}$ treatment with or without $300 \mathrm{mM} \mathrm{NaCl}$ in the light. After the initial illumination period, stomata of all plant lines were open (Supplemental Figure S8B). After the salt treatment, stomata became closed compared to control conditions (Figure 7A and 7B). However, stomata of Compl-S31D-5 rosette leaves were less affected by the salt treatment and their stomata remained about $40 \%$ more open when compared to other lines (Figure 7A and 7B). As drought and salt-stress induced ABA signaling is involved in stomatal closure, the plant lines were checked for stomatal closure in response to ABA. Leaf epidermal peels of 5 to 6 week-old Col-0, Compl-SWT-5, Compl-S31D-5 and Compl-S31A-7 plants were prepared in darkness at the end of the night period. They were first incubated in stomatal opening buffer under a light for $4 \mathrm{~h}$ without $\mathrm{ABA}$, and then incubated for a further $4 \mathrm{~h}$ in the presence of $50 \mu \mathrm{m}$ ABA. Stomatal apertures were measured before and after ABA addition. In the absence of $\mathrm{ABA}$, stomata were open in all lines (Figure 7C and 7D) while after ABA treatment, stomata aperture was significantly decreased however Compl-S31D-5 stomata were less sensitive to ABA since their closure was reduced (by 40\%) when compared to other lines (Figure 7C and 7D). These observations suggest that ABA-induced stomatal closure was impaired in Compl-S31D-5 and this could help explain the differences seen between plant lines after a salt-stress.

\section{Compl-S31D plants have altered ABA-induced gene expression under salt treatment}

Since Compl-S31D lines were more sensitive to drought and salt stresses and they exhibited a reduced response to $\mathrm{NaCl}$-induced and $\mathrm{ABA}$-induced stomatal closure, it was decided to further explore the ABA signaling responses of the plant lines. Therefore, the expression of several ABA-responsive and ABA-biosynthesis genes was examined by qPCR, before and after a $6 \mathrm{~h}$ salt treatment $(50 \mathrm{mM})$ of 2-week-old in vitro grown Col-0, Compl-SWT-5, ComplS31A-7 and Compl-S31D-5 seedlings. The expression of the ABA-responsive genes COR15A 
(Wilhelm and Thomshow, 1993), RAB18 (Lang and Palva, 1992), RD22 (YamaguchiShinozaki and Shinozaki, 1993b), RD29A (Lee et al., 2016), RD29B (Nordin et al., 1993) and P5CS1 (Ábrahám et al., 2003) and of the ABA-biosynthesis gene NCED3 (Iuchi et al., 2001) increased after the salt treatment in all lines (Figure 8). However, there was a trend for all transgenic lines to show a slightly lower salt-induced expression of RD29B, COR15A and NCED3 while this was significant for the salt-induced expression of $R A B 18$ and $R D 22$. However, salt treatment led to only a low induction of P5CS1 in Compl-S31D-5 (2-fold) when compared to Col-0, Compl-SWT-5 and Compl-S31A-7 (10-fold) while RD29A expression in Compl-S31D-5 was induced more (8-fold) than in Col-0, Compl-SWT-5 and Compl-S31A-7 (5-fold) (Figure 8).

\section{Discussion}

\section{Reduced SHMT activity of shm 1-1 complemented lines negatively impacts} photosynthetic $\mathrm{CO}_{2}$ assimilation and plant growth

In order to evaluate the impact of SHMT1 S31 phosphorylation on plant metabolism and growth, the shm1-1 mutant was complemented with SHMT1-S31D (phospho-mimetic), SHMT1-S31A (non-phosphorylatable) or SHMT1-SWT (non-mutated) forms (Figure 2A). In air-grown conditions, each recombinant SHMT1 form reversed the shm1-1 photorespiratory growth phenotype however they remained smaller than wild-type Col-0 plants (Figure 2B, 3A and 3B). This observation was associated with a 50\% lower SHMT activity in all complemented rosettes compared to Col-0 controls and correlated with reduced SHMT1 protein levels (Figure 2C, 2D and Supplemental Figure S2). Therefore, if the phosphomimetic SHMT1 S31 is indeed equivalent to phosphorylation, it did not appear to regulate maximal SHMT1 activity. As might be expected, the reduced SHMT activity plants also exhibited lower photosynthetic $\mathrm{CO}_{2}$ assimilation rates when compared with Col-0 (Supplemental Table S1). This suggested that under standard air-grown conditions, SHMT1 activity had become limiting for the photorespiratory cycle requirements of the transgenic plants, probably leading to toxic $2 \mathrm{PG}$ accumulation and/or limited C-recycling that negatively impact Calvin Cycle activity and $\mathrm{CO}_{2}$ assimilation. Indeed, an inhibition of photosynthesis is a common feature of a blocked photorespiratory cycle as seen by transferring photorespiratory mutants, including shm1-1, from high $\mathrm{CO}_{2}$ conditions to air (Somerville and Ogren, 1981; Chastain and Ogren, 1989; Timm et al., 2012). The photorespiratory nature of the observed 
growth phenotype of our complemented lines was confirmed by the absence of any growth phenotype under high $\mathrm{CO}_{2}$ conditions (Figure 3C and 3D). The non-optimal SHMT1 activity also had an impact on rosette metabolite levels in a similar manner (albeit less important) to those reported for shml-1 when transferred from high to normal $\mathrm{CO}_{2}$ air (Kuhn et al., 2013). Major changes were observed in several photorespiratory metabolite levels, as well as certain sugars, amino and organic acids. Indeed, the 5-to-6 fold increase in glycine is a good indicator of the limiting SHMT1 photorespiratory activity in the complemented lines. The higher serine levels might be due to alternative serine biosynthesis pathways such as the phosphorylated pathway and the glycerate pathway (Ros et al., 2014). Glucose and fructose increased (Figure 5) and this could reflect altered starch metabolism as recently reported in PGLP1 knock-down lines (Flügel et al., 2017) to allow the production of soluble sugars for cell metabolism when photosynthesis is impacted by an inefficient photorespiratory cycle. Surprisingly, the limiting SHMT1 activity had only small effects on amino and organic acid levels although proline content was reduced while $\alpha$-ketoglutarate amounts were increased (as already reported by Kuhn et al., 2013). Both of these metabolites are associated with glutamate metabolism, however glutamate levels only increased by $40 \%$ while glutamine increased by $70-90 \%$ thus suggesting a limited impact of reduced SHMT1 activity on GS/GOGAT cycle functioning and nitrogen assimilation.

The sensitivity of Compl-S31D lines to salt stress in air-grown conditions is due to a further reduction of SHMT1 protein and activity

Previous studies have shown shmtl mutants to be more sensitive to $\mathrm{NaCl}$ stress (Moreno et al., 2005; Zhou et al., 2012). Interestingly, Compl-S31D lines were more sensitive to salt stress, showing reduced growth and yellow rosettes as well as a lower FW and shorter primary root growth compared to the other lines when grown in air (Figure 3 and S3A). No difference was observed between the complemented lines in response to mannitol-generated osmotic stress. Indeed, transgenic lines remained smaller than Col-0 even though plant growth was severely reduced compared to control conditions (Figure 3A, 3B). Interestingly, this phenotype disappeared when plants were grown under high- $\mathrm{CO}_{2}$ conditions (Figure 3C, 3D and S3B) thus showing that the salt stress phenotype of Compl-S31D lines was linked to photorespiration. Since the transgenic lines had only 50\% of normal Col-0 SHMT activity and already exhibited a growth phenotype in air, it was possible that the additional salt-effect on Compl-S31D lines reflected a further inhibition of SHMT1 activity. This hypothesis appeared 
to be correct since there was a further decrease in SHMT activity (of about 43\%) in ComplS31D-5 seedlings when salt-stressed compared to control conditions while no significant differences were observed for Compl-SWT-5 and Compl-S31A-7 lines (Figure 4A and Table S2). This decrease in activity correlated with a further reduction in SHMT protein (of about $35 \%$ ) (Figure 4B, C). So after a salt stress, Compl-S31D rosettes contained only $33 \%$ of WT Col-0 SHMT activity (Figure 4A) and exhibited a more severe growth inhibition phenotype compared to the other lines (Figure 3, S2 and S3). This suggested that a critical SHMT1 activity had been reached that no longer allowed the plants to cope with a salt stress-induced increase of photorespiration. Again, it should be noted that transgenic plants with only 50\% SHMT activity already showed a small growth phenotype in non-stressed air-grown conditions. The need to reduce a photorespiratory enzyme activity below a certain level before observing a phenotype is common. Rice glycolate oxidase (GOX) activity had to be reduced below $60 \%$ to observe reduced photosynthetic activity (Xu et al., 2009). Arabidopsis goxl-1 showed no growth phenotype with only $27 \%$ of WT leaf GOX activity, while a photorespiratory phenotype required the activity to be reduced to a residual 5-10\% (Dellero et al., 2016). So, we conclude that the salt-induced sensitivity phenotype of Compl-S31D lines was due to their low photorespiratory SHMT activity brought about by a salt-induced decrease in SHMT1 protein abundance. It was recently proposed that during salt stress, UBP16-dependent SHMT1 stability reduced the accumulation of reactive oxygen species thus increasing the activity of a Na$/ \mathrm{H}^{+}$antiporter (Zhou et al., 2012). Therefore, it is possible that a low SHMT1 activity could reduce cell $\mathrm{Na}^{+}$export leading to salt stress sensitivity. Perhaps, the action of UBP16 is altered by phospho-mimetic SHMT1-S31 in Compl-S31D lines thus leading to decreased protein stability under salt conditions. In Arabidopsis, ICP55 removes the N-terminal S30 of "mature" SHMT1 thus making it less stable due to an increased turnover rate (Huang et al., 2015). It was found that S30 was correctly removed from SHMT1 in Compl-SWT-5 and Compl-S31A-7 rosettes but not in the Compl-S31D-5 line (Supplemental Figure S1). Surprisingly, SHMT1 S31D protein abundance appeared to be similar to SHMT1 S31A and SHMT SWT proteins in normal growth conditions (soil and in vitro) while it decreased in response to salt stress (Figure 2C, 4B, 4C and Supplemental Figure S2). Thus, the presence of S30-containing SHMT1 S31D protein did not appear to impact SHMT1 stability under non-stress conditions. Further evidence suggests that the N-terminal sequence of SHMT1 is important for stability since a deletion of the first six or more amino acids of sheep SHMT decreased the heat-stability of its oligomeric structure (Jagath et al., 1997). 
Perhaps the additional negative charges of a phosphate (or an aspartate) at the $\mathrm{N}$-terminus of the protein could result in a less stable SHMT1 under salt stress conditions.

\section{Decreased SHMT1 activity in phospho-mimetic Compl-S31D alters metabolic responses to salt stress}

In response to salt stress, a number of metabolite levels were altered in all plant lines studied including decreases in glutamine, asparagine, tryptophan, $\alpha$-ketoglutarate, glucose and fructose while serine and myo-inositol increased (Figure 5). The limited number of metabolic changes suggested that our salt treatment was rather mild. Globally, the SHMT1 transgenic lines that maintained $50 \%$ of WT Col-0 SHMT activity underwent similar salt-stress metabolic rearrangements as WT Col-0 plants thus indicating that at this low SHMT1 activity additional adverse affects on plant metabolism where not induced by a $50 \mathrm{mM} \mathrm{NaCl}$ treatment. This was perhaps unexpected since salt stress led to stomatal closure (Figure 7A, 7B) that should increase photorespiration and a requirement for a high SHMT1 activity. Therefore, it was surprising to observe a salt-stress induced decrease of glycine (Figure 5) that was negatively correlated with SHMT activity (Figure 4A) suggesting that glycine was being mobilized for other processes such as the biosynthesis of glutathione, an important stressinduced molecule involved in ROS-detoxification (Noctor et al., 2012). Indeed, a trend was observed of increased metabolite levels associated with stress responses including proline and myo-inositol while galactaric acid increased in all lines except Compl-S31D-5 (Figure 5). The latter has been reported to have a role in plants under stress conditions (Perlikowski et al., 2016; Sprenger et al., 2018), proline is an essential osmoprotectant in response to salt stress (Szabados and Savouré, 2009) and myo-inositol is important for plant tolerance to drought and salt stresses (Kaur et al., 2013). The reduced levels of these three metabolites in saltstressed Compl-S31D-5 plants might partially explain their increased sensitivity to salt and drought stresses. Compl-S31D-5 rosettes were also different compared to other lines with respect to polyamine accumulation (Figure 5) while ornithine also accumulated in Compl$S 31 D-5$ in response to a $\mathrm{NaCl}$ treatment and citrulline remained at a higher level compared to other lines (Figure 5). Therefore, salt-stressed Compl-S31D-5 plants appeared to modify their metabolism to favor polyamines. The reason for this is unknown but it might reflect the biosynthesis of nitrogen-rich compounds at a lower carbon cost due to limited carbon resources arising from an inability to maintain sufficient photorespiratory carbon recycling and a further reduced photosynthetic capacity. 
561

562

563

564

565

566

567

568

569

570

571

572

573

574

575

576

577

578

579

580

581

582

583

584

585

586

587

588

589

590

591

592

Compl-S31D plants were not only more sensitive to salt stress but also to drought stress recovery while Compl-S31D detached rosettes exhibited a higher and faster water loss compared to the other lines (Supplemental Figure S6A and S6B). The water loss phenotype was not due to a defect in initial water content (Supplemental Figure S6C) and it probably reflected the altered stress-induced stomatal movements of Compl-S31D lines (Figure 7). Indeed, both salt and drought stresses lead to stomatal closure to reduce transpiratory water losses however the reduction of leaf internal $\mathrm{CO}_{2}$ levels should reduce photosynthesis while increasing photorespiration (Chastain et al., 2014; Fricke et al., 2004). By studying photorespiratory mutants, including $s h m 1$, it was recently shown that photorespiration is important for stomatal movements (Eisenhut et al., 2017). Since no differences in stomatal conductance or aperture were detected between our SHMT1 lines in air-grown conditions (Figure 7 and Supplemental Table S1), it appeared that 50\% of WT Col-0 SHMT1 activity was enough to maintain stomatal aperture. However, after a salt stress or an ABA treatment that led to stomatal closure, the stomata of Compl-S31D-5 remained more open (Figure 7) while SHMT activity was further reduced to only $35 \%$ of the Col-0 value. This decreased stomatal closure in response to salt and to ABA (Figure 7) could impact the sensitivity of Compl-S31D-5 to salt stress and drought recovery. Since mesophyll organic acid metabolism is involved in regulating stomatal aperture (Araújo et al., 2011), metabolic changes in Compl$S 31 D$ rosettes might help explain the observed differences. However, malate and fumarate levels were not significantly different between Compl-S31D and WT Col-0 lines (Figure 5 and Supplemental Table S3). The plant hormone, ABA, is involved in stomatal closure during both salt and drought stresses and Compl-S31D plants were less sensitive to ABA (Figure 7C, 7D). This is not the first time that photorespiration has been implicated in ABA signaling. A screen of EMS-mutagenized plants for an altered expression of the $\mathrm{ABA}$ and $\mathrm{NaCl}$ induced gene RD29A (Responsive to Dessication 29A) led to the identification of two glutamate:glyoxylate transferase 1 (GGT1) alleles (Verslues et al., 2007). However, while ggt1 mutants were ABA desensitized due to a basal $\mathrm{H}_{2} \mathrm{O}_{2}$ accumulation, this was not the case for Compl-S31D lines as their rosettes contained similar amounts of $\mathrm{H}_{2} \mathrm{O}_{2}$ when compared to the other lines under control conditions and only a small $20 \%$ difference in rosette $\mathrm{H}_{2} \mathrm{O}_{2}$ levels was measured in response to salt stress (Figure 6 and Supplemental Figure S5). To examine whether ABA signaling was involved in the differential stomatal responses, the expression of several stress marker genes, RD29A, RD29B, RAB18, RD22, COR15A and P5CS1, was tested 
in response to $\mathrm{NaCl}$ treatment. $R D 29 B, R A B 18, R D 22$ and $C O R 15 A$ expressions are regulated by ABA while RD29A expression is regulated by $\mathrm{ABA}$ and by an unknown pathway activated by $\mathrm{NaCl}$ stress ( Lang and Palva, 1992; Sakuma et al., 2002; Wilhelm and Thomshow, 1993; Yamaguchi-Shinozaki and Shinozaki, 1993a; Yamaguchi-Shinozaki and Shinozaki, 1993b) and salt-induced P5CS1 expression can involve either ABA or an ABA-independent phospholipase C (PLC) pathway (Strizhov et al., 1997; Parre et al., 2007). Since RD29A, $R D 29 B, R A B 18, R D 22$ and $C O R 15 A$ were induced in response to our $\mathrm{NaCl}$ treatment in all lines (Figure 8), the ABA signaling pathway appeared unaffected at the whole rosette level. Moreover, ABA biosynthesis does not seem to be affected either as the expression of NCED3, encoding a rate-limiting enzyme for stress-induced ABA synthesis (Iuchi et al, 2001; Tan et al, 2003), did not differ between the lines either in control conditions or in response to salt stress (Figure 8). However, a possible role of the PLC pathway was suggested by the lower salt stress induction of P5CS1 in Compl-S31D-5 (Figure 8).

In conclusion, we have shown that photorespiration is an important metabolic process when plants undergo salt stress. Indeed, the absence/reduction of photorespiration brought about by high $\mathrm{CO}_{2}$ conditions almost abolished the salt-induced growth defects. In air, plants must maintain an efficient photorespiratory cycle flux since we observed that a low SHMT1 activity increased salt stress sensitivity. With respect to S31 phosphorylation of SHMT1, based on the position of S31 in the observed phosphopeptides, it is not involved in the removal of the mitochondrial signal peptide nor N-terminal processing by ICP55. Since the phospho-mimetic SHMT1 did not exhibit an altered maximal SHMT activity, S31 phosphorylation does not appear to be directly involved in modulating enzymatic activity. However, the stability of S31 phospho-mimetic SHMT1 appeared to be reduced under mild salt stress conditions, although we would not expect SHMT1 S31 phosphorylation to be induced under such conditions due to its detrimental effect. Finally, photorespiratory cycle flux efficiency appears to influence stomatal movements in a yet to be discovered mechanism that could involve a common metabolic component involved in stomatal closure.

\section{Acknowledgements}

This work was supported by a public grant overseen by the French National Research Agency (ANR) as part of the «Investissement d'Avenir» program, through the "Lidex-3P" project and a French State grant (ANR-10-LABX-0040-SPS) funded by the IDEX Paris-Saclay, ANR-11- 
625 IDEX-0003-02. This work was also supported by the ANR-14-CE19-0015 grant REGUL3P 626 awarded to MH. YL was supported by a PhD fellowship from the China Scholarship Council. 627 We thank Andreas Weber (University of Dusseldorf, Germany) for kindly providing the 628 shm1-1 mutant and Hermann Bauwe (University of Rostock, Germany) for the anti-SHMT 629 antibodies. We thank Sophie Filleur and Julie Hodin for their help with stomatal aperture 630 experiments. The present work benefited from the light microscopy facility of Imagerie-Gif, 631 supported by "France-BioImaging" (ANR-10-INBS-04-01), and the Labex "Saclay Plant 632 Science" (ANR-11-IDEX-0003-02). We thank Marlène Davanture and Michel Zivy of 633 PAPPSO, (http://pappso.inra.fr) for determining SHMT1 N-terminal sequences. No conflict 634 of interest is declared. 


\section{References}

Abadie, C., Mainguet, S., Davanture, M., Hodges, M., Zivy, M., and Tcherkez, G. (2016). Concerted changes in the phosphoproteome and metabolome under different $\mathrm{CO}_{2} / \mathrm{O}_{2}$ gaseous conditions in Arabidopsis rosettes. Plant and Cell Physiology, 57, 1544-1556.

Ábrahám, E., Rigó, G., Székely, G., Nagy, R., Koncz, C., and Szabados, L. (2003). Lightdependent induction of proline biosynthesis by abscisic acid and salt stress is inhibited by brassinosteroid in Arabidopsis. Plant Molecular Biology, 51, 363-372.

Anderson, L. (1971). Chloroplast and cytoplasmic enzymes. II. Pea leaf triose phosphate isomerases. Biochimica et Biophysica Acta, 1, 237-244.

Araújo, W. L., Nunes-Nesi, A., Osorio, S., Usadel, B., Fuentes, D., Nagy, R., Balbo, I., Lehmann, M., Studart-Witkowski, C., Tohge, T., et al. (2011). Antisense inhibition of the iron-sulphur subunit of succinate dehydrogenase enhances photosynthesis and growth in tomato via an organic acid-mediated effect on stomatal aperture. Plant Cell, 23, 600627.

Bauwe, H., Hagemann, M., and Fernie, A. R. (2010). Photorespiration: players, partners and origin. Trends in Plant Science, 15, 330-336.

Bloom, A. J. (2015). Photorespiration and nitrate assimilation: A major intersection between plant carbon and nitrogen. Photosynthesis Research, 123, 117-128.

Boex-Fontvieille, E., Daventure, M., Jossier, M., Zivy, M., Hodges, M., and Tcherkez, G. (2013). Photosynthetic control of Arabidopsis leaf cytoplasmic translation initiation by protein phosphorylation. PLoS one, 8, e70692.

Camejo, D., Romero-Puertas, M. del C., Rodríguez-Serrano, M., Sandalio, L. M., Lázaro, J. J., Jiménez, A., and Sevilla, F. (2013). Salinity-induced changes in S-nitrosylation of pea mitochondrial proteins. Journal of Proteomics, 79, 87-99.

Chastain, C. J., and Ogren, W. L. (1989). Glyoxylate inhibition of ribulose bisphosphate carboxylase oxygenase activation state in vivo. Plant and Cell Physiology, 30, 937-944.

Chastain, D. R., Snider, J. L., Collins, G. D., Perry, C. D., Whitaker, J., and Byrd, S. A. (2014). Water deficit in field-grown Gossypium hirsutum primarily limits net photosynthesis by decreasing stomatal conductance, increasing photorespiration, and increasing the ratio of dark respiration to gross photosynthesis. Journal of Plant Physiology, 171, 1576-1585.

Chung, E., Cho, C.-W., So, H.-A., Kang, J.-S., Chung, Y. S., and Lee, J.-H. (2013). Overexpression of $\operatorname{VrUBC} 1$, a mung bean E2 ubiquitin-conjugating enzyme, enhances osmotic tolerance in Arabidopsis. PLoS One, 8, e66056.

Clough, S. J., and Bent, A. F. (1998). Floral dip : a simplified method for Agrobacteriummediated transformation of Arabidopsis thaliana. The Plant Journal, 16, 735-743.

Cui, L., Lu, Y., Li, Y., Yang, C., and Peng, X. (2016). Overexpression of glycolate oxidase confers improved photosynthesis under high light and high temperature in rice. Frontiers in Plant Science, 7, 1-12.

Dellero, Y., Lamothe-Sibold, M., Jossier, M., and Hodges, M. (2015). Arabidopsis thaliana 
ggt1 photorespiratory mutants maintain leaf carbon/nitrogen balance by reducing RuBisCO content and plant growth. The Plant Journal, 83, 1005-1018.

Dellero, Y., Jossier, M., Glab, N., Oury, C., Tcherkez, G., and Hodges, M. (2016). Decreased glycolate oxidase activity leads to altered carbon allocation and leaf senescence after a transfer from high $\mathrm{CO}_{2}$ to ambient air in Arabidopsis thaliana. Journal of Experimental Botany, 67, 3149-3163.

Edwards, K., Johnstone, C., and Thompson, C. (1991). A simple and rapid method for the preparation of plant genomic DNA for PCR analysis. Nucleic Acids Research, 19, 1991.

Eisenhut, M., Bräutigam, A., Timm, S., Florian, A., Tohge, T., Fernie, A. R., Bauwe, H., and Weber, A. P. M. (2017). Photorespiration is crucial for dynamic response of photosynthetic metabolism and stomatal movement to altered $\mathrm{CO}_{2}$ availability. Molecular Plant, 10, 47-61.

Engel, N., Ewald, R., Gupta, K. J., Zrenner, R., Hagemann, M., and Bauwe, H. (2011). The presequence of Arabidopsis serine hydroxymethyltransferase SHM2 selectively prevents import into mesophyll mitochondria. Plant Physiology, 157, 1711-1720.

Fernie, A. R., Aharoni, A., Willmitzer, L., Stitt, M., Tohge, T., Kopka, J., Caroll, A. J., Saito, K., Fraser, P. D., and DeLuca, V. (2011). Recommendations for reporting metabolite data. Plant Cell, 23, 2477-2482.

Fiehn, O., Wohlgemuth, G., Scholz, M., Kind, T., Lee D.Y., Lu, Y., Moon, S., and Nikolau, B. (2008). Quality control for plant metabolomics: reporting MSI-compliant studies. The Plant Journal, 53, 691-704.

Flügel, F., Timm, S., Arrivault, S., Florian, A., Stitt, M., Fernie, A. R., and Bauwe, H. (2017). The photorespiratory metabolite 2-phosphoglycolate regulates photosynthesis and starch accumulation in Arabidopsis. Plant Cell, 29, 2537-2551.

Fricke, W., Akhiyarova, G., Veselov, D., and Kudoyarova, G. (2004). Rapid and tissuespecific changes in $\mathrm{ABA}$ and in growth rate in response to salinity in barley leaves. Journal of Experimental Botany, 55, 1115-1123.

Hanson, A. D., and Gregory, J. F. (2011). Folate biosynthesis, turnover, and transport in Plants. Annual Review of Plant Biology, 62, 105-125.

Huang, S., Nelson, C. J., Li, L., Taylor, N. L., Ströher, E., Petereit, J., and Millar, A. H. (2015). INTERMEDIATE CLEAVAGE PEPTIDASE 55 modifies enzyme amino termini and alters protein stability in Arabidopsis mitochondria. Plant Physiology, 168, $415-427$.

Iuchi, S., Kobayashi, M., Taji, T., Naramoto, M., Seki, M., Kato, T., Tabata, S., Kakubari, Y., Yamaguchi-Shinozaki, K., and Shinozaki, K. (2001) Regulationof Drought tolerance by gene manipulation of 9-cis-epoxycarotenoid dioxygenase, a key enzyle in abscisic acid biosynthesis in Arabidopsis. The Plant Journal, 4, 325-333.

Jagath, J. R., Sharma, B., Bhaskar, B., Datta, A., Rao, N. A., and Savithri, H. S. (1997). Importance of the amino terminus in maintenance of oligomeric structure of sheep liver cytosolic serine hydroxymethyltransferase. European Journal of Biochemistry, 247, 372379. 
Jossier, M., Kroniewicz, L., Dalmas, F., Le Thiec, D., Ephritikhine, G., Thomine, S., BarbierBrygoo, H., Vavaseur, A., Filleur, S., and Leonhardt, N. (2010). The Arabidopsis vacuolar anion transporter, AtCLCc, is involved in the regulation of stomatal movements and contribute to salt tolerance. The Plant Journal, 64, 563-576.

Kaur, H., Verma, P., Petla, B. P., Rao, V., Saxena, S. C., and Majee, M. (2013). Ectopic expression of the ABA-inducible dehydration-responsive chickpea 1-myo-inositol 1phosphate synthase 2 (CaMIPS2) in Arabidopsis enhances tolerance to salinity and dehydration stress. Planta, 237, 321-335.

Kelly, G. J., and Latzko, E. (1976). Inhibition of spinach-leaf phosphofructokinase by 2phosphoglycollate. FEBS Letters, 68, 55-58.

Kopka, J., Schauer, N., Krueger, S., Birkemeyer, C., Usadel, B., Bergmüller, E., Dörmann, P., Weckwerth, W., Gibon, Y., Stitt, M., Willmitzer, L., Fernie, A.R., and Steinhauser, D. (2005). GMD@CSB.DB: the Golm metabolome database. Bioinformatics, 21, 16351638.

Kuhn, A., Engqvist, M. K. M., Jansen, E. E. W., Weber, A. P. M., Jakobs, C., and Maurino, V. G. (2013). D-2-hydroxyglutarate metabolism is linked to photorespiration in the shm1-1 mutant. Plant Biology, 15, 776-784.

Lang, V., and Palva, E. T. (1992) The expression of a rab-related gene, $R A B 18$, is induced by abscisic acid during the cold acclimatation process of Arabidopsis thaliana (L.) Heynh. Plant Molecular Biology, 5, 951-962.

Laemmli UK (1970). Cleavage of structural proteins during the assembly of the head of bacteriophage T4. Nature, 227, 680-685.

Lee, S. Y., Boon, N. J., Webb, A. A. R., and Tanaka, R. J. (2016). Synergistic activation of $R D 29 A$ via integration of salinity stress and abscisic acid in Arabidopsis thaliana. Plant and Cell Physiology, 57, 2147-2160.

Li, J., and Hu, J. (2015). Using co-expression analysis and stress-based screens to uncover Arabidopsis peroxisomal proteins involved in drought response. PLoS One, 10, e0137762.

Marchand, C., Le Maréchal, P., Meyer, Y., Miginiac-Maslow, M., Issakidis-Bourguet, E., Decottignies, P., Balmer, Y., Vensel, W. H., Tanaka, C. K., Hurkman, W. J., et al. (2004). Thioredoxin links redox to the regulation of fundamental processes of plant mitochondria. Proteomics, 4, 2696-2706.

McClung, C. R., Hsu, M., Painter, J. E., Gagne, J. M., Karlsberg, S. D., and Salomé, P. a. (2000). Integrated temporal regulation of the photorespiratory pathway. Circadian regulation of two Arabidopsis genes encoding serine hydroxymethyltransferase. Plant Physiology, 123, 381-392.

Merlot, S., Leonhardt, N., Fenzi, F., Valon, C., Costa, M., Piette, L., Vavasseur, A., Genty, B., Boivin, K., Müller, A., et al. (2007). Constitutive activation of a plasma membrane $\mathrm{H}^{+}$-ATPase prevents abscisic acid-mediated stomatal closure. The EMBO Journal, 26, 3216-3226.

Mishra, P., Mishra, V., Takabe, T., Rai, V., and Singh, N. K. (2016). Elucidation of salttolerance metabolic pathways in contrasting rice genotypes and their segregating 
progenies. Plant Cell Reports, 35, 1273-1286.

Moreno, J. I., Martín, R., and Castresana, C. (2005). Arabidopsis SHMT1, a serine hydroxymethyltransferase that functions in the photorespiratory pathway influences resistance to biotic and abiotic stress. The Plant Journal, 41, 451-463.

Noctor, G., Bergot, G., Mauve, C., Thominet, D., Lelarge-Trouverie, C., and Prioul, J. L. (2007). A comparative study of amino acid measurement in leaf extracts by gas chromatography-time of flight-mass spectrometry and high performance liquid chromatography with fluorescence detection. Metabolomics, 3, 161-174.

Noctor, G., Mhamdi, A., Chaouch, S., Han, Y., Neukermans, J., Marquez-Garcia, B., Queval, G., and Foyer, C. (2012). Glutathione in plants: an integrated overview. Plant, Cell \& Environment, 35, 454-484.

Noctor, G., Lelarge-Trouverie, C., and Mhamdi, A. (2015). The metabolomics of oxidative stress. Phytochemistry, 112, 33-53.

Nordin, K., Vahala, T., and Palva, E. T. (1993). Differential expression of two related, lowtemperature-induced genes in Arabidopsis thaliana (L.) Heynh. Plant Molecular Biology, 21, 641-653.

Parre, E., Ghars, M. A., Leprince, A.-S., Thiery, L., Lefebvre, D., Bordenave, M., Richard, L., Mazars, C., Abdelly, C., and Savoure, A. (2007). Calcium signaling via phospholipase C is essential for proline accumulation upon ionic but not nonionic hyperosmotic stresses in Arabidopsis. Plant Physiology, 144, 503-512.

Perlikowski, D., Czyżniejewski, M., Marczak, Ł., Augustyniak, A., and Kosmala, A. (2016). Water deficit affects primary metabolism differently in two Lolium multiflorum/Festuca arundinacea introgression forms with a distinct capacity for photosynthesis and membrane regeneration. Frontiers in Plant Science, 7, 1-16.

Ros, R., Munoz-Bertomeu, J., and Stephan Krueger (2014). Serine in plants: Biosynthesis, metabolism, and functions. Trends in Plant Science, 19, 564-569.

Sakuma, Y., Liu, Q., Dubouzet, J. G., Abe, H., Yamaguchi-Shinozaki, K., and Shinozaki, K. (2002). DNA-binding specificity of the ERF/AP2 domain of Arabidopsis DREBs, transcription factors involved in dehydration- and cold-inducible gene expression. Biochemical and Biophysical Research Communications, 290, 998-1009.

Schauer, N., Steinhauser, D., Strelkov, S., Schomburg, D., Allison, G., Moritz, T., Lundgren, K., Roessner-Tunali, U., Forbes, M.G., Willmitzer, L., Fernie, A.R., Kopka, J. (2005). GC-MS libraries for the rapid identification of metabolites in complex biological samples. FEBS Letters, 6, 1332-1337.

Simic, P., Willuhn, J., Sahm, H., and Eggeling, L. (2002). Identification of GlyA (encoding serine hydroxymethyltransferase) and its use together with the exporter ThrE to increase L-threonine accumulation by Corynebacterium glutamicum. Applied and Environmental Microbiology, 68, 3321-3327.

Somerville, C. R., and Ogren, W. L. (1981). Photorespiration deficient mutants of Arabidopsis thaliana lacking mitochrondrial serine transhydroxymethylase activity. Plant Physiology, 67, 666-671. 
Sørhagen, K., Laxa, M., Peterhänsel, C., and Reumann, S. (2013). The emerging role of photorespiration and non-photorespiratory peroxisomal metabolism in pathogen defence. Plant Biology, 15, 723-736.

Sprenger, H., Erban, A., Seddig, S., Rudack, K., Thalhammer, A., Le, M. Q., Walther, D., Zuther, E., Köhl, K. I., Kopka, J., et al. (2018). Metabolite and transcript markers for the prediction of potato drought tolerance. Plant Biotechnology Jorunal, 16, 939-950.

Strizhov, N., Abraham, E., Okresz, L., Blickling, S., Zilberstein, A., Schell, J., Koncz, C., and Szabados, L. (1997). Differential expression of two P5CS genes controlling proline accumulation during salt-stress requires $\mathrm{ABA}$ and is regulated by $\mathrm{ABA} 1, \mathrm{ABI} 1$ and AXR2 in Arabidopsis. The Plant Journal, 12, 557-569.

Szabados, L., and Savouré, A. (2009). Proline: a multifunctional amino acid. Trends in Plant Science, 15, 89-97.

Takahashi, S., Bauwe, H., and Badger, M. (2007). Impairment of the photorespiratory pathway accelerates photoinhibition of photosystem II by suppression of repair but not acceleration of damage processes in Arabidopsis. Plant Physiology, 144, 487-494.

Tan, B.-C., Joseph, L. M., Deng, W.-T., Liu, L., Li, Q.-B., Cline, K., and McCarty, D. R. (2003). Molecular characterization of the Arabidopsis 9-cis epoxycarotenoid dioxygenase gene family. The Plant Journal, 35, 44-56.

Tcherkez, G., Bligny, R., Gout, E., Mahe, A., Hodges, M., and Cornic, G. (2008). Respiratory metabolism of illuminated leaves depends on $\mathrm{CO}_{2}$ and $\mathrm{O}_{2}$ conditions. Proceedings of the National Academy of Sciences, 105, 797-802.

Timm, S., and Bauwe, H. (2013). The variety of photorespiratory phenotypes - employing the current status for future research directions on photorespiration. Plant Biology, 15, 737747.

Timm, S., Mielewczik, M., Florian, A., Frankenbach, S., Dreissen, A., Hocken, N., Fernie, A. R., Walter, A., and Bauwe, H. (2012). High-to-low $\mathrm{CO}_{2}$ acclimation reveals plasticity of the photorespiratory pathway and indicates regulatory links to cellular metabolism of Arabidopsis. PLoS One, 7, e42809.

Verslues, P. E., Kim, Y. S., and Zhu, J. K. (2007). Altered ABA, proline and hydrogen peroxide in an Arabidopsis glutamate:glyoxylate aminotransferase mutant. Plant Molecular Biology, 64, 205-217.

Voll, L. M., Jamai, A., Renné, P., Voll, H., McClung, C. R., and Weber, A. P. M. (2006). The photorespiratory Arabidopsis shm1 mutant is deficient in SHM1. Plant Physiology, 140, 59-66.

Waditee-Sirisattha, R., Sittipol, D., Tanaka, Y., and Takabe, T. (2012). Overexpression of serine hydroxymethyltransferase from halotolerant cyanobacterium in Escherichia coli results in increased accumulation of choline precursors and enhanced salinity tolerance. FEMS Microbiology Letters, 333, 46-53.

Waditee-Sirisattha, R., Kageyama, H., Tanaka, Y., Fukaya, M., and Takabe, T. (2016). Overexpression of halophilic serine hydroxymethyltransferase in fresh water cyanobacterium Synechococcus elongatus PCC7942 results in increased enzyme activities of serine biosynthetic pathways and enhanced salinity tolerance. Archives of 
Wilhelm, K.S., and Thomashow, M. F. (1993) Arabidopsis thaliana COR15B, an apparent homologue of COR15A, is strongly responsive to cold and ABA, but not drought. Plant Molecular Biology, 5, 1073-1077.

Xu, H., Zhang, J., Zeng, J., Jiang, L., Liu, E., Peng, C., He, Z., and Peng, X. (2009). Inducible antisense suppression of glycolate oxidase reveals its strong regulation over photosynthesis in rice. Journal of Experimental Botany, 60, 1799-1809.

Xu, Y. P., Yang, J., and Cai, X. Z. (2018). Glycolate oxidase gene family in Nicotiana benthamiana: genome-wide identification and functional analyses in disease resistance. Scientific Reports, 8, 8615.

Yamaguchi-Shinozaki, K., and Shinozaki, K. (1993a). Characterization of the expression of a desiccation-responsive RD29 gene of Arabidopsis thaliana and analysis of its promoter in transgenic plants. Molecular and General Genetics, 236, 331-340.

Yamaguchi-Shinozaki, K., and Shinozaki, K. (1993b). The plant hormone abscissic acid mediates the drought-induced expression but not the seed-specific expression of $R D 22$, a gene responsive to dehydratation stress in Arabidopsis thaliana. Molecular and General Genetics, 238, 17-25.

Zhou, H., Zhao, J., Yang, Y., Chen, C., Liu, Y., Jin, X., Chen, L., Li, X., Deng, X. W., Schumaker, K. S., et al. (2012). UBIQUITIN-SPECIFIC PROTEASE16 modulates salt yolerance in Arabidopsis by regulating $\mathrm{Na}^{+} / \mathrm{H}^{+}$antiport activity and serine hydroxymethyltransferase stability. Plant Cell, 24, 5106-5122.

\section{Figure legends}

Figure 1. A simplified scheme of the photorespiratory cycle with SHMT1 highlighted.

In a mitochondrion, the serine hydroxymethyl transferase 1 (SHMT1) catalyzes the conversion of glycine and 5,10-methylene-tetrahydrofolate $\left(5,10-\mathrm{CH}_{2}-\mathrm{THF}\right)$, produced by the glycine decarboxylase complex (GDC), to serine and THF. Abbreviation: RuBisCO, Ribulose-1,5-Bisphosphate Carboxylase/Oxygenase.

Figure 2. Characterization of shm1-1 complemented lines Compl-SWT, Compl-S31A and Compl-S31D.

(A) Schematic diagram of T-DNA organization and location of site-directed mutagenesis positions in the SHMT1 sequence. Conversions of phospho-S31 codon (TCT) to alanine (GCT) and aspartic acid (GAT) in Arabidopsis SHMT1 wild-type cDNA were performed by oligonucleotide-directed mutagenesis. Mutated nucleotides are underlined. RB and LB, right and left T-DNA border regions; SHMT1-Pro, SHMT1 promoter region sequence; T35S, cauliflower mosaic virus $35 \mathrm{~S}$ terminator; Hyg, hygromycin resistance gene. (B) Growth 
phenotype of Col-0 (control), shm1-1 mutant and complemented lines (Compl-SWT, ComplS31A, Compl-S31D) grown on soil in ambient air $\left(400 \mu \mathrm{L} \cdot \mathrm{L}^{-1} \mathrm{CO}_{2}\right)$. The photograph was taken after a 4-week growth period under short-day conditions. (C) and (D) Col-0 (control), shm1-1 mutant and complemented lines (Compl-SWT, Compl-S31A, Compl-S31D) were grown in short day and in high- $\mathrm{CO}_{2}$ condition for 4-weeks. Total soluble proteins were extracted from rosette leaves to analysis SHMT1 protein level (C) and SHMT activity (D). SHMT1 protein level was analyzed by western-blot using potato SHMT1 antibodies and actin was used as a loading control. SHMT activity was assayed by following the production of glycine by HPLC. Values are means \pm SD $(n=3$ independent experiments including at least 5 plants for each experiment). Statistical significance was determined by a Student's $t$-test $(\mathrm{P}<$ $0.05)$.

Figure 3. Salt sensitivity of air-grown complemented lines is reduced under high- $\mathrm{CO}_{2}$ growth conditions.

Growth phenotype of Col-0, Compl-SWT-5, Compl-S31D-5 and Compl-S31A-7 seedlings in response to salt stress. Seedlings were grown for 1 week on vertical $1 / 2 \mathrm{MS}$ plates and transferred to new $1 / 2 \mathrm{MS}$ medium with or without either $50 \mathrm{mM} \mathrm{NaCl}$ or $100 \mathrm{mM}$ mannitol for a further 21 or 25 days. (A) Air-grown seedlings, (C) $\mathrm{HC}$ grown $\left(3000 \mu \mathrm{L} \mathrm{CO}_{2} \cdot \mu \mathrm{L}^{-1}\right)$ seedlings. (B) and (D) show the FW values of seedlings shown in (A) and (C), respectively. Values are means \pm SD ( $\mathrm{n}>10$ seedlings). All experiments were repeated three times. Statistical significance was determined by a Student's $t$-test $(* \mathrm{P}<0.05 ; * * * \mathrm{P}<0.001)$.

Figure 4. SHMT1 protein stability is altered in Compl-S31D plants in response to salt stress.

Seven-day-old in vitro grown seedlings of Col-0, Compl-SWT-5, Compl-S31D-5 and ComplS31A-7 were transferred to $1 / 2 \mathrm{MS}$ plates with or without $50 \mathrm{mM} \mathrm{NaCl}$ for 3 weeks, then leaf total soluble proteins were extracted. (A) Leaf SHMT activity was measured by following glycine using an HPLC-based method. Values are means \pm SD $(n=3$ independent experiments). (B) Leaf SHMT1 protein abundance was determined by western-blot analyses using potato SHMT1 antibodies. Coomassie blue stained RuBisCO large subunit was used as a protein loading control. (C) SHMT1 and RuBisCO large-subunit signal intensities were quantified using Image J software. SHMT1 protein levels were normalized to RuBisCO largesubunit amounts, and relative protein levels of Compl-SWT-5 without $\mathrm{NaCl}$ treatment were set to 1 . Values are means \pm SD $(n=3$ independent experiments). Statistical significance was 
determined by a Student's $t$-test. Asterisks indicate significant differences compared to Col-0 under the same condition $(* \mathrm{P}<0.05)$; black triangles indicate significant differences compared with the same genotype $(\Delta \mathrm{P}<0.05)$.

\section{Figure 5. Metabolic response to salt stress of Compl-S31D line differs from Col-0,} Compl-SWT-5 and Compl-S31A-7 lines.

Plants were grown as described in Figure $2 \mathrm{~A}$ on $1 / 2 \mathrm{MS}$ with or without $50 \mathrm{mM} \mathrm{NaCl}$. Relative metabolite contents (mean $\pm \mathrm{SD}, \mathrm{n}=4$ independent samples) are given for whole rosette leaves of Col-0 (black), Compl-SWT-5 (white), Compl-S31D-5 (light grey), Compl-S31A-7 (dark grey) with the corresponding Col-0 control content (without $\mathrm{NaCl}$ ) set to 1 . Histograms show metabolites that were significantly different in control $(1 / 2 \mathrm{MS})$ and $\mathrm{NaCl}(\mathrm{NaCl})$ conditions in Compl-SWT-5, Compl-S31D-5 or Compl-S31A-7 lines compared to Col-0 under the same condition (marked with an asterisk *) or compared with the initial value of the same genotype (marked with $\boldsymbol{\Delta})$. Statistical significance was determined by a Student's $t$-test $(\mathrm{P}<0.05)$. See also Table S3.

\section{Figure 6. Salt stress induced $\mathrm{H}_{2} \mathrm{O}_{2}$ levels.}

Two-week-old Col-0, Compl-SWT-5, Compl-S31D-5, and Compl-S31A-7 seedlings were treated with or without $150 \mathrm{mM} \mathrm{NaCl}$ for $14 \mathrm{~h}$, and $\mathrm{H}_{2} \mathrm{O}_{2}$ levels were (A) observed by DAB staining and (B) quantified using a fluorescent assay. Values are means \pm SD $(n=3$ independent experiments). Statistical significance was determined by a Student's $t$-test $(* \mathrm{P}<0.05)$.

\section{Figure 7. Compl-S31D-5 stomata show an altered closure response to ABA and salt} treatments.

Five-week-old plants (Col-0, Compl-SWT-5, Compl-S31D-5 and Compl-S31A-7) were pretreated under light for $2.5 \mathrm{~h}$ to induce stomatal opening and then they were watered with or without $300 \mathrm{mM} \mathrm{NaCl}$ for $4 \mathrm{~h}$. Stomatal apertures of epidermal peels were measured before and after the $\mathrm{NaCl}$ treatment. (A) Micrographs of $\mathrm{NaCl}$-induced stomatal closure of $\mathrm{Col}-0$, Compl-SWT-5, Compl-S31D-5 and Compl-S31A-7. (B) Measured stomatal aperture size (pore width/length) with or without $\mathrm{NaCl}$ treatment. Values are means $\pm \mathrm{SD}(\mathrm{n}>60)$. Experiments were repeated at least three times. Statistical significance was determined by a Student's $t$-test ( $\mathrm{P}<0.05)$. Leaf epidermal strips of Col-0, Compl-SWT-5, Compl-S31D-5 and Compl-S31A-7 were incubated in stomatal opening buffer under light for $4 \mathrm{~h}$ and then further incubated in the 
same buffer containing $50 \mu \mathrm{M}$ ABA for another $4 \mathrm{~h}$. Stomatal pore areas were measured

946 before and after ABA treatment. (C) Micrographs of ABA-induced stomatal closure of Col-0,

947 Compl-SWT-5, Compl-S31D-5 and Compl-S31A-7. (D) Measured stomatal pore area before or 948 after ABA treatment. Values are means \pm SD $(n>60)$ and experiments were repeated at least 949 three times. Statistical significance was determined by a Student's $t$-test $(\mathrm{P}<0.05)$.

950

951 Figure 8. Relative expression levels of several ABA-responsive genes in Compl-SWT-5, 952 Compl-S31D-5 and Compl-S31A-7 in response to salt treatment.

953 Two-week-old Arabidopsis seedlings were transferred to liquid medium lacking or containing $95450 \mathrm{mM} \mathrm{NaCl}$ for $6 \mathrm{~h}$. Gene expression levels were measured by quantitative RT-PCR. Values 955 are means $\pm \mathrm{SD}$ ( $\mathrm{n}=3$ independent experiments). Actin2 was used as an internal control, and 956 expression levels were normalized to Col-0 without salt treatment. P5CS1, Pyrroline-5957 carboxylate synthase 1; RD29A, Responsive to desiccation 29A; RD29B, Responsive to 958 desiccation 29B; $R A B 18$, Responsive to ABA 18; RD22, Responsive to desiccation 22; 959 COR15A, Cold-regulated 15A; NCED3, 9-cis epoxycarotenoïd dioxygenase 3. Statistical 960 significance was determined by a Student's $t$-test and asterisks indicate significant difference 961 compared to the control line for each condition $(* \mathrm{P}<0.05)$. 


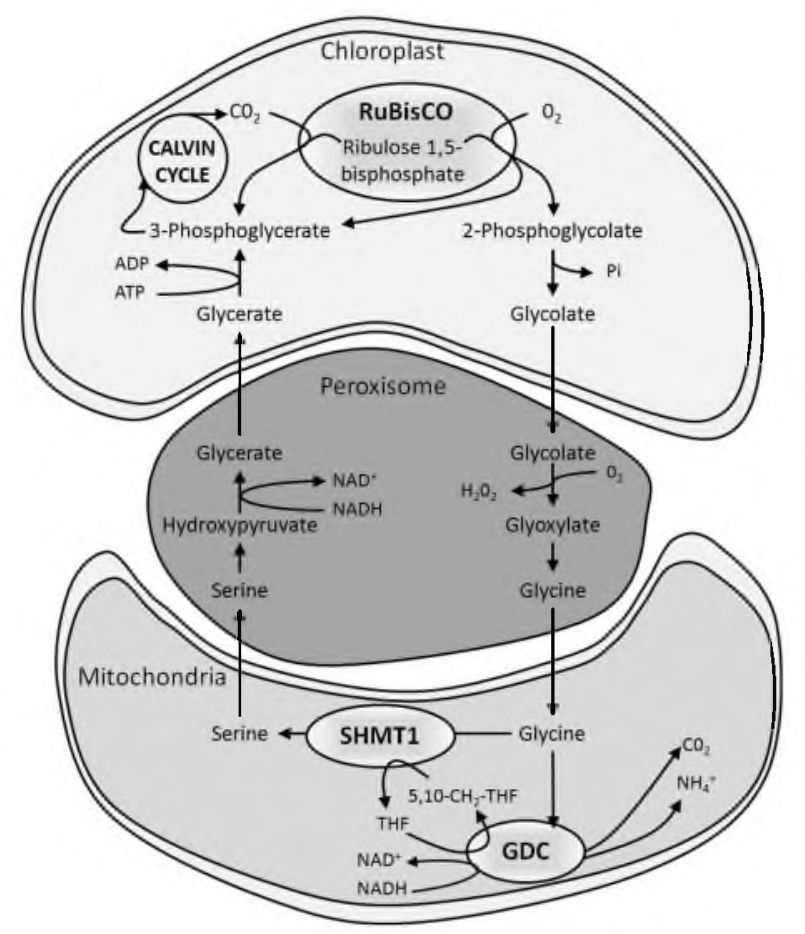


A

SHMT1-Pro SHMT1 CDNA

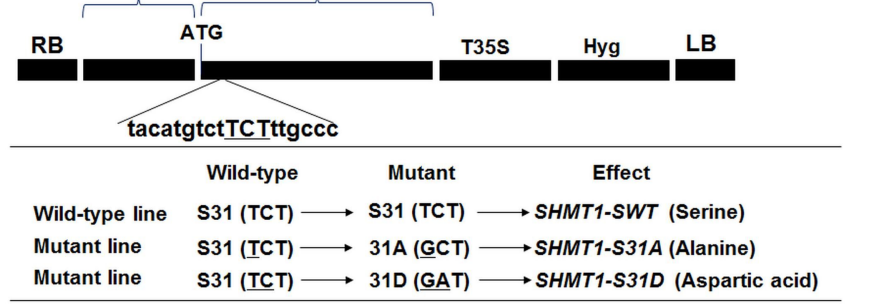

B

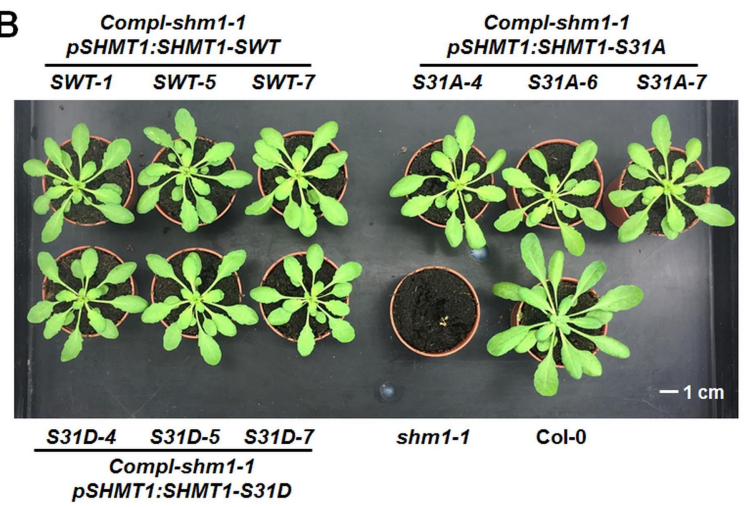

C

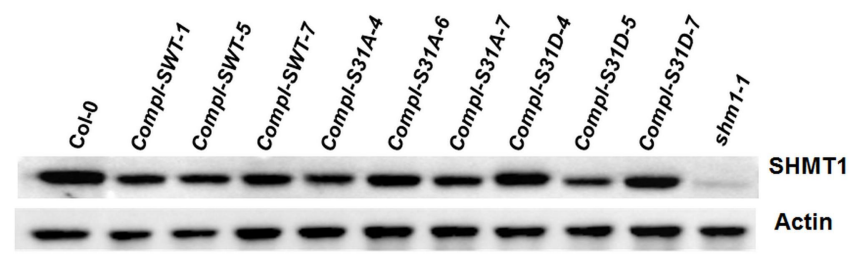

D

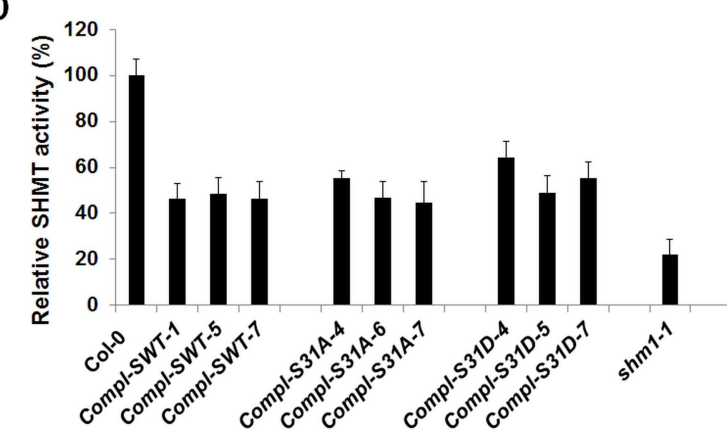


A

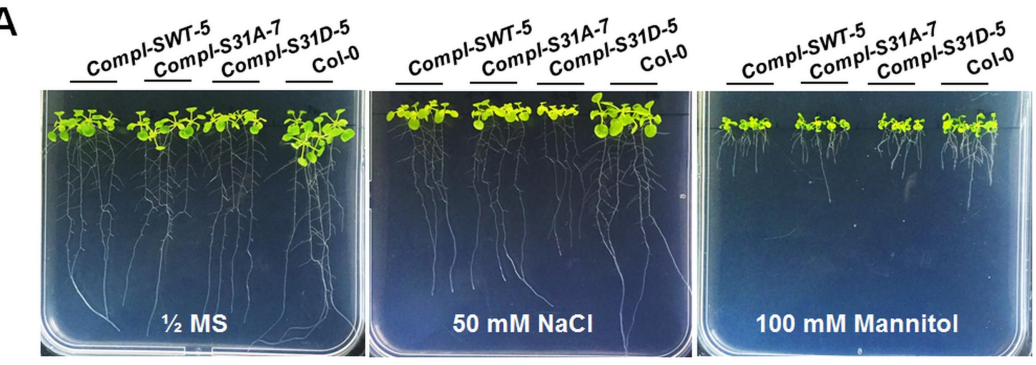

C

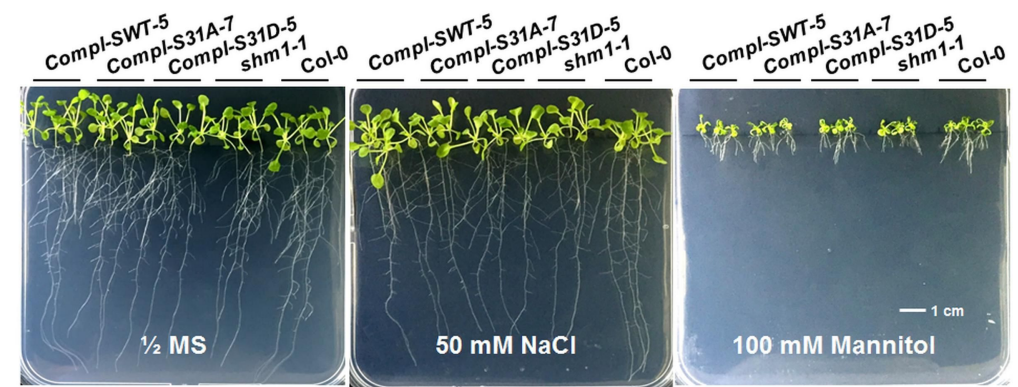

B

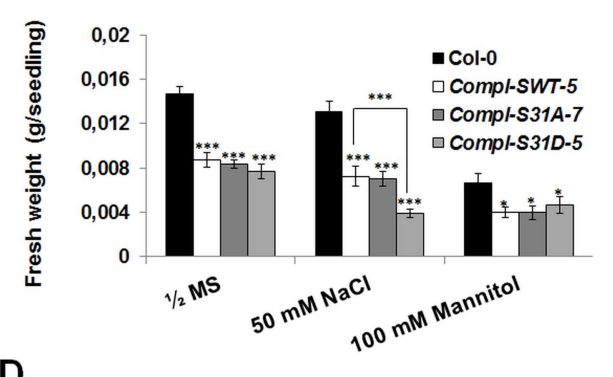

D

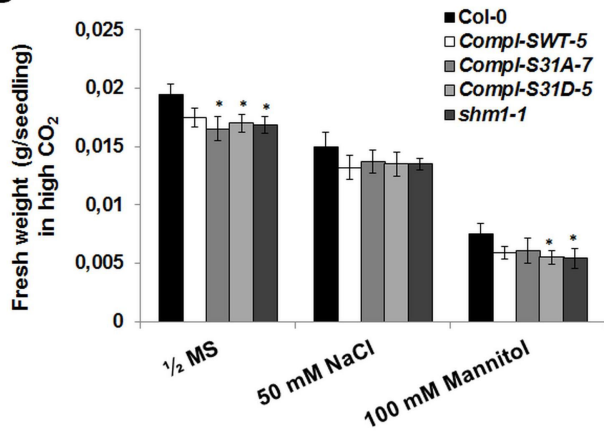




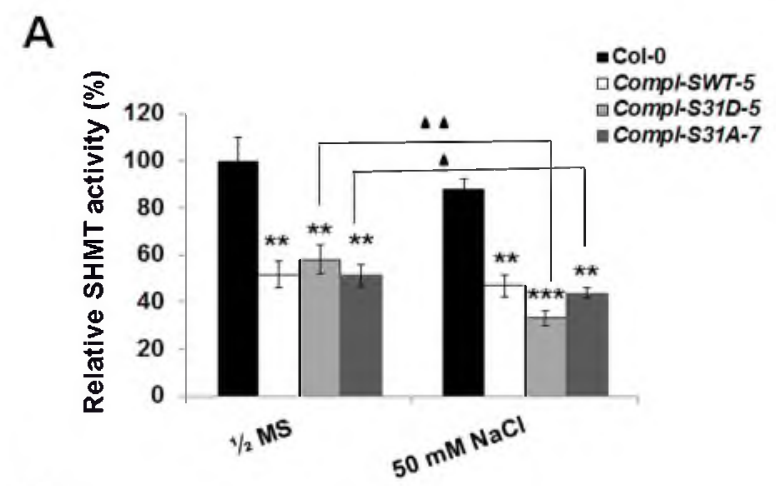

B

$\frac{1 / 2 M S}{\text { SWT-5 S31D-5 S31A-7 SWT-5 S31D-5 S31A-7 }}$
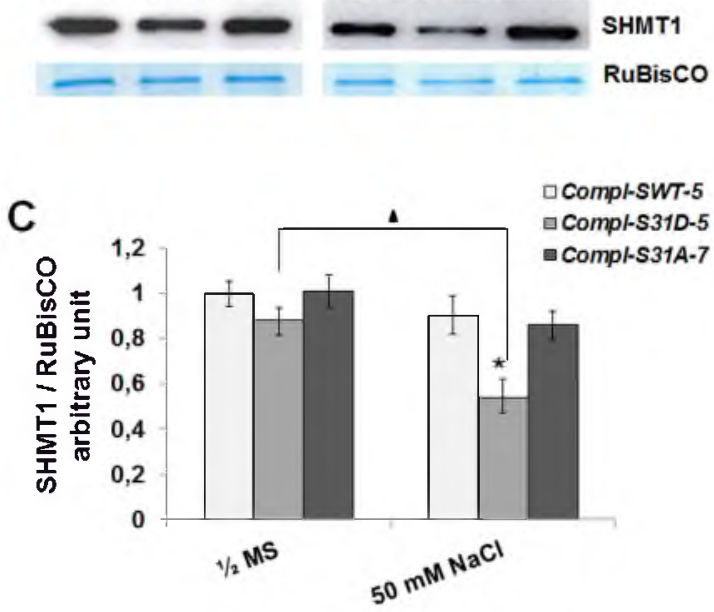
Amino acids
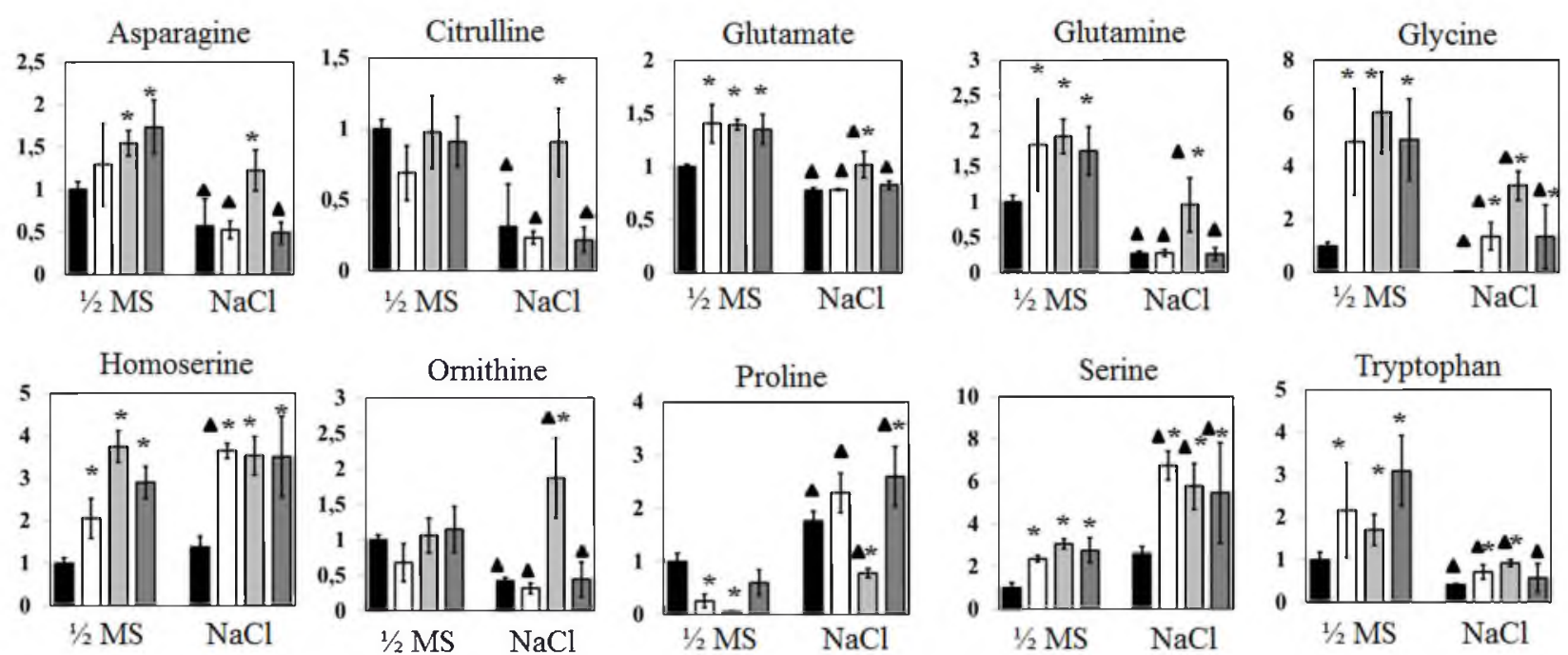

Organic acids
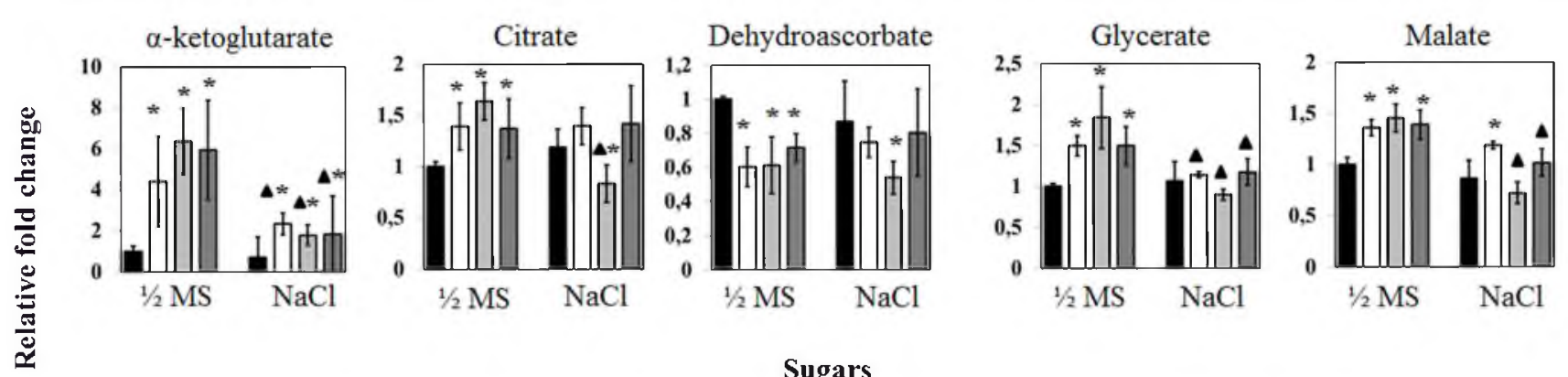

Sugars
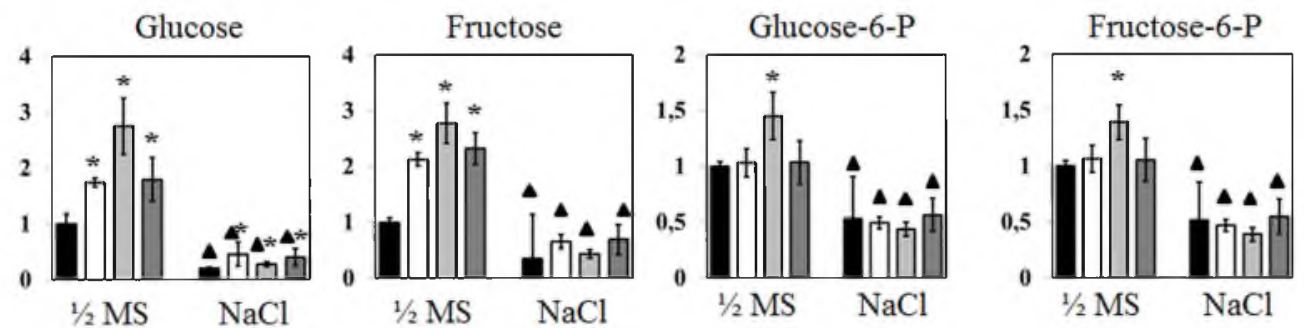

Miscellaneous
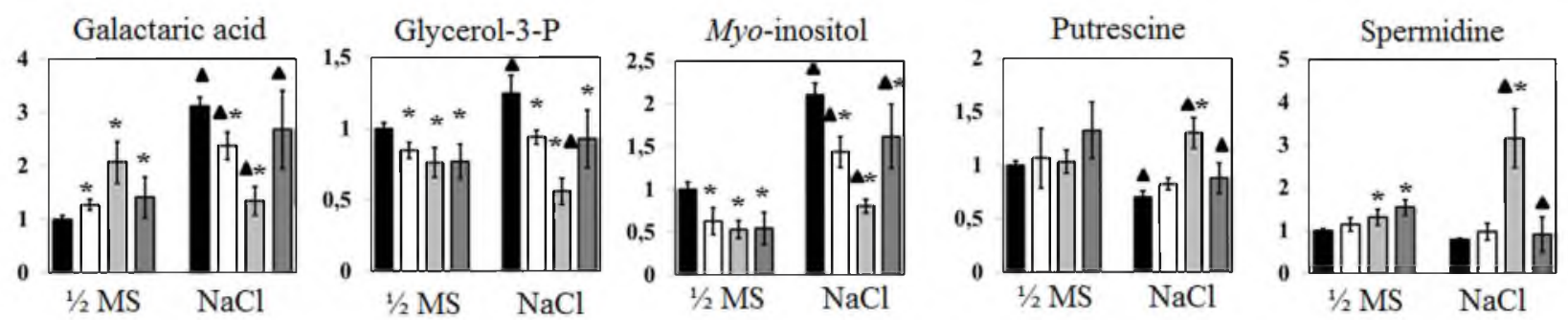

Col-0 $\square$ Compl-SWT-5 $\square$ Compl-S31D-5 $\square$ Compl-S31A-7 


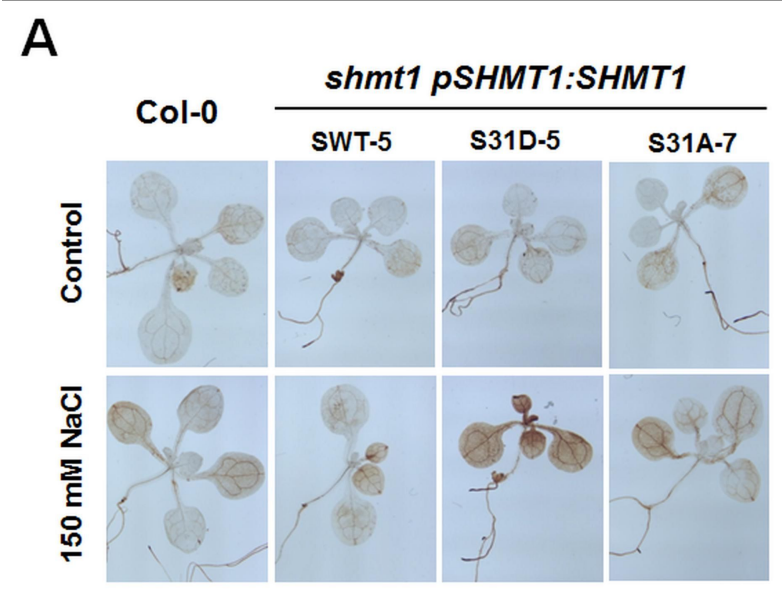

B

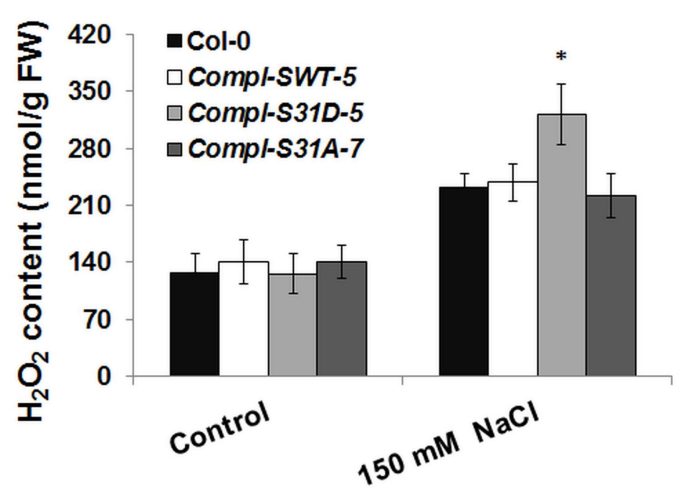


A

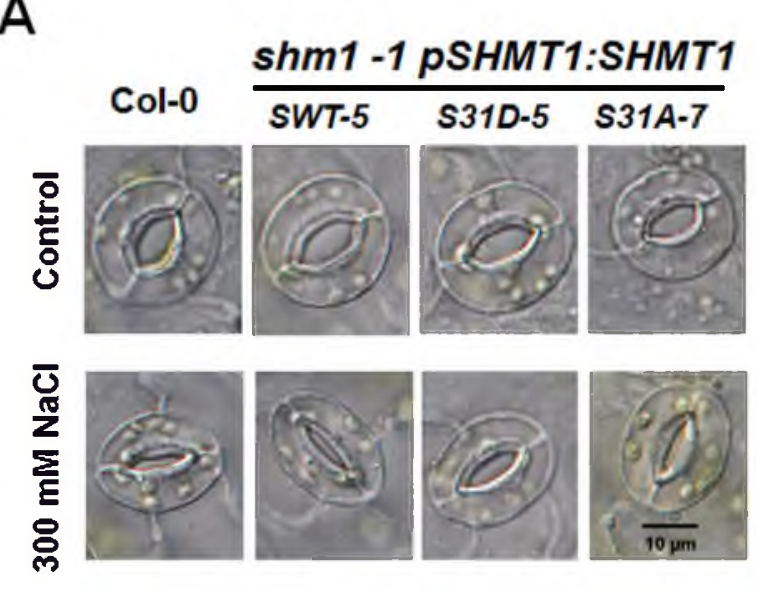

B

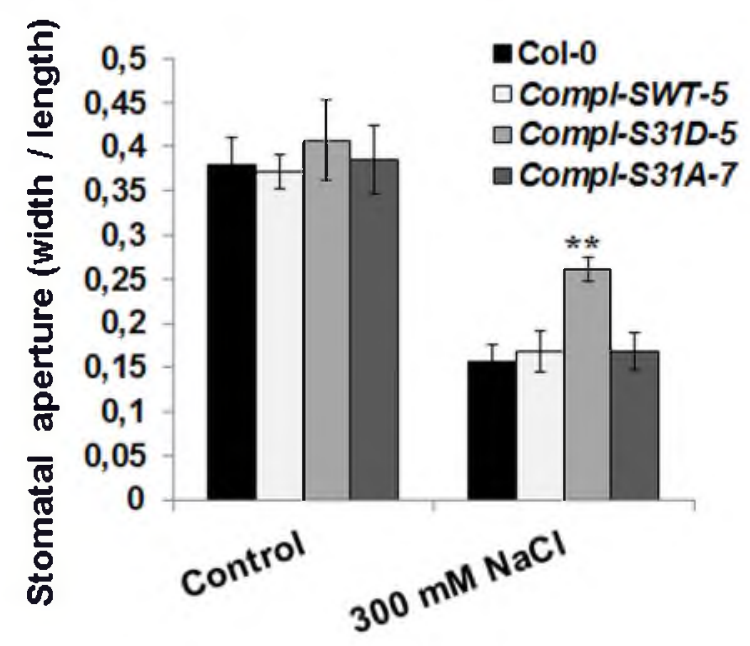

C

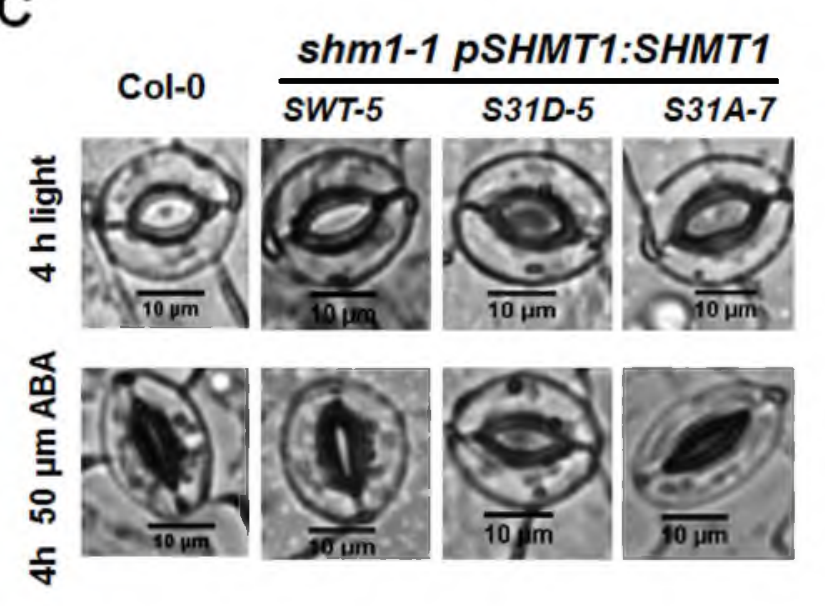

D

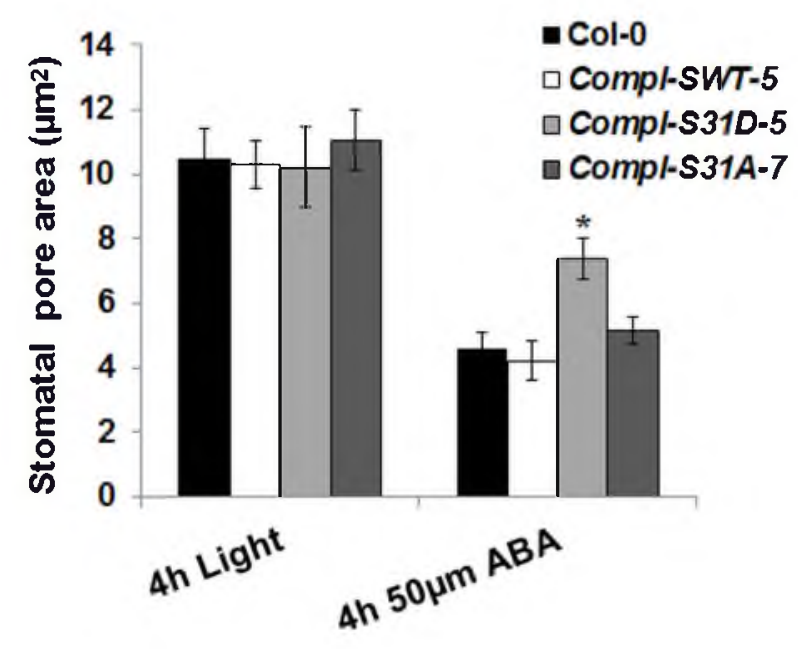



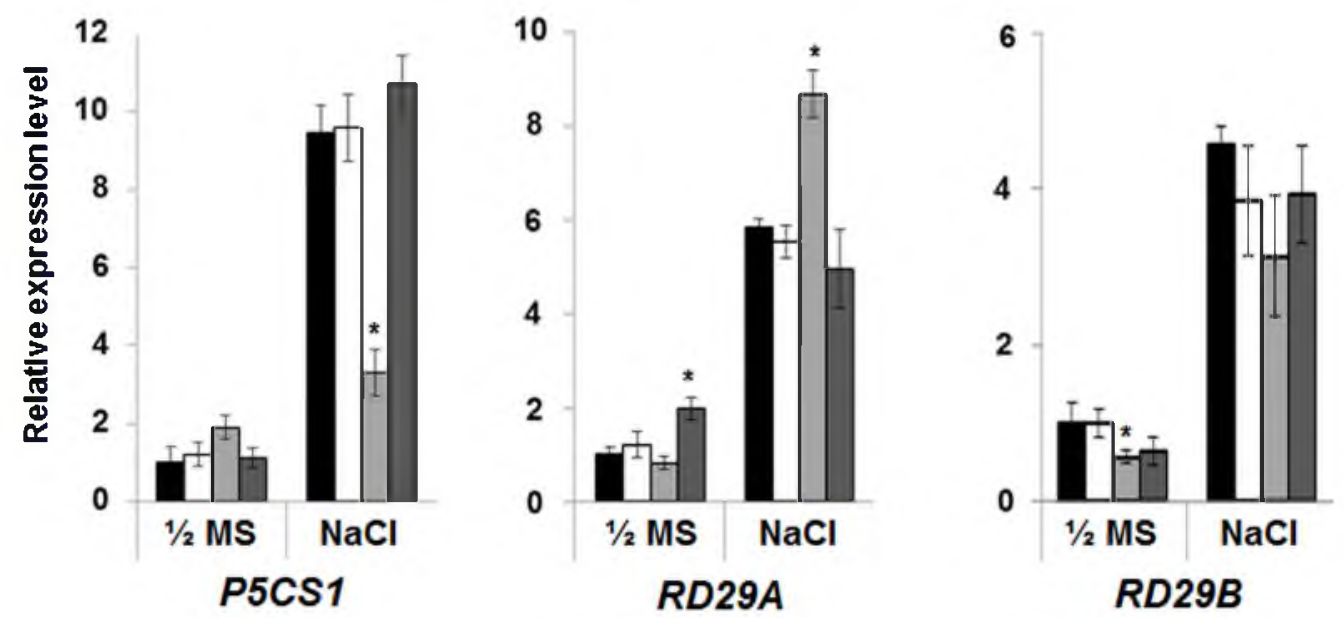

4
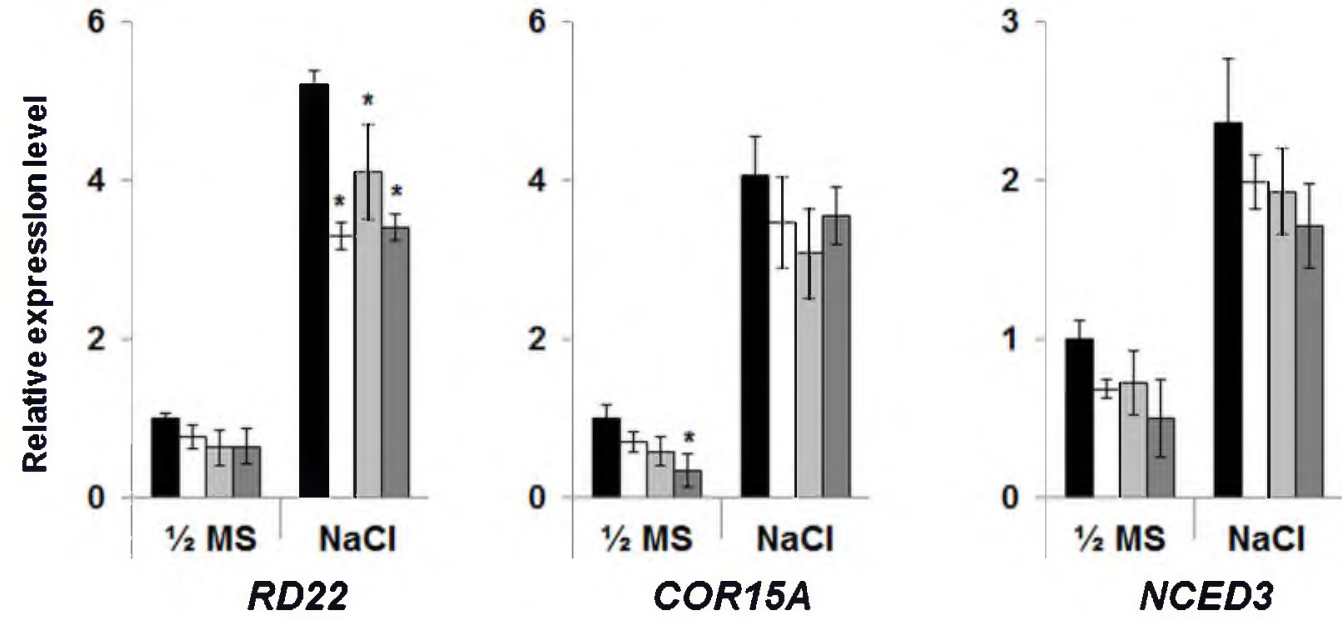

2

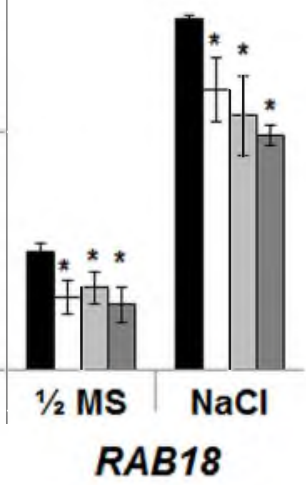

-Col-0

$\square$ Compl-SWT-5

口Compl-S31D-5

-Compl-S31A-7 